OPEN ACCESS

Edited by:

Gang Li,

South China Sea Institute

of Oceanology, Chinese Academy

of Sciences (CAS), China

Reviewed by:

Zhijian Jiang,

South China Sea Institute

of Oceanology, Chinese Academy

of Sciences (CAS), China

Amrit Kumar Mishra,

Indian Institute of Technology

Bhubaneswar, India

*Correspondence:

Pedro Beca-carretero pbeca@iim.csic.es

Specialty section:

This article was submitted to Global Change and the Future Ocean,

a section of the journal

Frontiers in Marine Science

Received: 26 June 2021

Accepted: 16 August 2021

Published: 28 September 2021

Citation:

Beca-Carretero $P$,

Azcárate-García T, Julia-Miralles M,

Stanschewski CS, Guihéneuf $F$ and

Stengel DB (2021) Seasonal

Acclimation Modulates the Impacts of

Simulated Warming and Light

Reduction on Temperate Seagrass

Productivity and Biochemical

Composition

Front. Mar. Sci. 8:731152

doi: 10.3389/fmars.2021.731152

\section{Seasonal Acclimation Modulates the Impacts of Simulated Warming and Light Reduction on Temperate Seagrass Productivity and Biochemical Composition}

\author{
Pedro Beca-Carretero ${ }^{1,2 *}$, Tomás Azcárate-García ${ }^{1,3}$, Marc Julia-Miralles ${ }^{4}$, \\ Clara S. Stanschewski ${ }^{5}$, Freddy Guihéneuf ${ }^{6}$ and Dagmar B. Stenge ${ }^{1}$
}

${ }^{1}$ Botany and Plant Science, School of Natural Sciences, National University of Ireland Galway, Galway, Ireland, ${ }^{2}$ Department of Oceanography, Instituto de Investigacións Mariñas (IIM-CSIC), Vigo, Spain, ${ }^{3}$ Departamento de Biología, Área de Ecología, Facultad de Ciencias del Mar y Ambientales, Universidad de Cádiz, Cádiz, Spain, ${ }^{4}$ Posgrado en Oceanografia Costera, Facultad de Ciencias Marinas, Universidad Autónoma de Baja California, Ensenada, Mexico, ${ }^{5}$ King Abdullah University of Science and Technology, Center for Desert Agriculture, Biological and Environmental Sciences and Engineering Division, Thuwal, Saudi Arabia, ${ }^{6}$ SAS Inalve, Villefranche-sur-Mer, France

Increases in seawater temperature and reduction in light quality have emerged as some of the most important threats to marine coastal communities including seagrass ecosystems. Temperate seagrasses, including Zostera marina, typically have pronounced seasonal cycles which modulate seagrass growth, physiology and reproductive effort. These marked temporal patterns can affect experimental seagrass responses to climate change effects depending on the seasons of the year in which the experiments are conducted. This study aimed at evaluating how seasonal acclimatization modulates productivity and biochemical responses of Zostera marina to experimental warming and irradiance reduction. Seagrass shoots were exposed to different temperatures $\left(6,12,16,20\right.$, and $24^{\circ} \mathrm{C}$ ), combined with high (180 $\mu \mathrm{mol}$ photons $\mathrm{m}^{-2} \mathrm{~s}^{-1}$ ) and low $\left(60 \mu \mathrm{mol}\right.$ photons $\mathrm{m}^{-2} \mathrm{~s}^{-1}$ ) light conditions across four seasons (spring: April, summer: July, and autumn: November 2015, and winter: January 2016). Plants exhibited similar temperature growth rates between 16 and $20^{\circ} \mathrm{C}$; at $24^{\circ} \mathrm{C}$, a drastic reduction in growth was observed; this was more accentuated in colder months and under low irradiance conditions. Higher leaf growth rates occurred in winter while the largest rhizomes were reached in experiments conducted in spring and summer. Increases in temperature induced a significant reduction in polyunsaturated fatty acids (PUFA), particularly omega-3 (n-3 PUFA). Our results highlight that temperate seagrass populations currently living under temperature limitation will be favored by future increases in sea surface temperature in terms of leaf and rhizome productivity. Together with results from this study on $Z$. marina from a temperate region, a wider review of the reported impacts of experimental warming indicates the likely reduction 
in some compounds of nutritional importance for higher trophic levels in seagrass leaves. Our results further demonstrate that data derived from laboratory-based studies investigating environmental stress on seagrass growth and acclimation, and their subsequent interpretation, are strongly influenced by seasonality and in situ conditions that precede any experimental exposure.

Keywords: seasonal acclimatization, temperature, irradiance, fatty acids, nutritional composition, Ireland, Zostera marina, reproductive effort

\section{HIGHLIGHTS}

- Seagrass responses to temperature and irradiance are partly modulated by seasonal acclimatization

- 3-4 ${ }^{\circ} \mathrm{C}$ above in situ temperatures will favor temperate seagrass growth and production

- Optimum growth temperature is similar across seasons and light levels

- Light limitation enhances high temperature stress

- Rises in temperature reduce the production and accumulation of omega-3 fatty acids.

\section{INTRODUCTION}

Seagrasses play a vital role in coastal zones in terms of productivity, support of biodiversity, constitution as a primary food source of nutrition for several marine organisms, protection of the coastline, carbon storage and nutrient retention (Hemminga and Duarte, 2000; Carruthers et al., 2007; Nordlund et al., 2018). However, over the last few decades, global seagrass distribution has been reduced by around $30 \%$. Particularly Zostera marina is lost at a rate of $1.3 \%$ per year due to anthropogenic pressures and climate change effects (Orth et al., 2006; Waycott et al., 2009). Noteworthy, a recent study revealed signs of recovery of European seagrass meadows due to conservation efforts (de los Santos et al., 2019).

Zostera marina L. is the dominant habitat-forming seagrass species in the northern hemisphere, currently distributed from subtropical regions in the Pacific coast of Mexico $\left(24.3^{\circ} \mathrm{N}\right)$ and in the Mediterranean Sea $\left(35.1^{\circ} \mathrm{N}\right)$, to sub-Arctic regions in Greenland $\left(64.2^{\circ} \mathrm{N}\right)$ and in the northern coast of Russia $\left(74.3^{\circ} \mathrm{N}\right.$ ) covering a latitudinal distribution of $\sim 50^{\circ}$ (Short et al., 2006). Reflecting its broad distribution, this species is adapted to a wide range of environmental conditions including annual average temperatures ranging from $\sim 1$ to $25^{\circ} \mathrm{C}$ (Tyberghein et al., 2012). Temperate seagrasses typically have pronounced seasonal cycles which modulate seagrass growth, physiology and flowering (e.g., Orth and Moore, 1986; Alcoverro et al., 2001). Temperate $Z$. marina populations usually grow faster and develop larger photosynthetic structures in summer months under more suitable climate conditions, storing high reserves of energetic compounds in the rhizomes such as carbohydrates. In less favorable environmental conditions, plants reduce their aboveground structures to reduce respiratory demands, and use the energetic reserves (e.g., Olesen and Sand-Jensen, 1993; Lee et al., 2005).
Temperature is one the most important environmental factors controlling seagrass physiological, growth and reproductive processes (Lee et al., 2007; Qin et al., 2020b). Global warming has emerged as an important threat for marine coastal species including seagrass ecosystems (e.g., Duarte, 2002). Seagrass responses to predicted increases in temperature, including Z. marina, depends on population-specific thermal tolerance and their capacity to adapt to new local climate conditions (Short and Neckles, 1999). As a result, expected global warming may drive different effects within $Z$. marina populations across their latitudinal distribution range. For instance, some subarctic populations were reported to exhibit more favorable responses to temperature increases (Krause-Jensen et al., 2015; Olesen et al., 2015; Beca-Carretero et al., 2018a). On the contrary, warming may negatively affect southern $Z$. marina populations when summer temperatures can exceed their physiological optima (e.g., Reusch et al., 2005; Lopez-Calderon et al., 2016). Physiological and biochemical responses of centrally distributed populations to warming events remain more unclear (Beca-Carretero et al., 2018a; Aoki et al., 2020; Dubois et al., 2020).

Irradiance is the most relevant environmental driver affecting photosynthetic activity and the vertical distribution of seagrasses (Ralph et al., 2007). The predicted increase in the frequency and intensity of storms alongside continuous coastal modifications will likely cause a reduction in underwater light and thus, cause low light stress in seagrass populations (Duarte, 2002; Silva et al., 2013; Bertelli and Unsworth, 2018). While some species can tolerate low light for short periods, longer exposure can cause chronic physiological stress and eventual seagrass loss (e.g., Short and Wyllie-Echeverria, 1996; Nguyen et al., 2021). Global warming is expected to enhance light limitation stress in seagrasses as minimum irradiance requirements, to maintain adequate growth and physiological performance, are increased at higher temperatures (Lee et al., 2007). Experimental studies of $Z$. marina populations distributed at their central distribution range reported that plants exposed to light-limited conditions (50 $\mu \mathrm{mol}$ photons $\mathrm{m}^{-2} \mathrm{~s}^{-1}$ ) were highly vulnerable to temperatures above $20^{\circ} \mathrm{C}$, causing growth inhibition and negative carbon budgets (Beca-Carretero et al., 2018b). Recently, in situ studies provide evidence for largescale declines in $Z$. marina meadows due to combined effects of light reduction due to precipitation event sand sediment runoff and anomalous warming episodes of temperatures above $28^{\circ} \mathrm{C}$ (Johnson et al., 2021).

Warming episodes are well documented for summer seasons, however, with the substantial change in climate in northern 
latitudes, they also occur during cold seasons in higher latitudes where Z. marina is present (Short et al., 2007; Bokhorst et al., 2009). Anomalous climate events throughout the year can alter seasonal rhythms, uncoupling plant internal clocks and, consequently, affecting their vegetative development, population dynamics and reproductive cycles (Alcoverro et al., 2001; Bokhorst et al., 2009; Bjerke et al., 2011). As a result, different growth and physiological seagrass responses to climate change effects can be expected depending on the seasons of the year in which these events occur. However, the majority of the controlled temperature exposure experiments using seagrasses were conducted within a single season (e.g., review in BecaCarretero et al., 2018a). Some studies reported differential sensitivities to temperature and irradiance treatments across seasons in the temperate species $Z$. marina (Staehr and Borum, 2011), which makes it highly relevant to assess the potential effects of warming during different seasons. While several studies have reported effects of warming or change in light climate on seagrass ecosystems (review in Koch et al., 2013; review in Roca et al., 2016; review in Nguyen et al., 2021), only few authors assessed the potential synergic effects of multiple stressors (e.g., Koch et al., 2007; Ontoria et al., 2019b).

Irish coasts are characterized by the presence of $Z$. marina meadows in subtidal areas reaching maximum depths of 4-6 m, and in lesser extension in the lower intertidal (Madden et al., 1993; Beca-Carretero et al., 2019b). In Ireland, there is an overall lack of seagrass research including their distribution, ecology and healthy status (Dale et al., 2007; Wilkes et al., 2017). Recent studies reported that large subtidal Z. marina meadows remain undisturbed along the Irish coast (Beca-Carretero et al., 2019b; Cott et al., 2021), and it is expected that their distribution is wider than previously known (Beca-Carretero et al., 2020; Hastings et al., 2020). Ireland's climate is defined as a temperate oceanic climate, characterized by defined climatic seasons with a lack of extreme cold or warm temperatures. Western Irish seagrasses are exposed to minimum temperatures of $4-5^{\circ} \mathrm{C}$ in winter and a maximum of $16-17^{\circ} \mathrm{C}$ reached in summer. With optimal temperature for growth of worldwide $Z$. marina populations ranging from 15.3 to $24^{\circ} \mathrm{C}$ (review in Lee et al., 2007; review in Beca-Carretero et al., 2018 b), it can be expected that an increase of $2-3^{\circ} \mathrm{C}$ in Irish nearshore systems will favor seagrasses growth and production (Beca-Carretero et al., 2019b).

Seagrasses constitute an important source of food for several marine organisms; important nutritional compounds include proteins, carbohydrates or lipids and fatty acids (review in Kim et al., 2021). Despite the ecological importance of seagrasses as a food source to higher tropic levels, only few studies have explicitly assessed the role of environmental changes on their nutritional value. Recently, studies of the seagrass species Z. marina, P. oceanica and Halophila stipulacea reported changes in essential nutritional compounds including reductions of polyunsaturated fatty acids (PUFAs) relative to saturated fatty acids (SFAs), lower leaf fiber and proteins, and an increase in leaf necrosis in response to increasing temperatures have been documented (e.g., Beca-Carretero et al., 2018b, 2020; Hernán et al., 2019; Nguyen et al., 2020a). Such biochemical changes render seagrasses exposed to stress conditions a less desirable food source for herbivores (Hernán et al., 2017) although it is currently not clear how warming can modify biochemical composition and associated nutritional value. Here, we assessed how combined temperature and irradiance exposure treatments affect centrally distributed $Z$. marina populations descriptors (morphological, production and biochemical composition), and how such responses are modulated by seasonal acclimatization. Specifically, we investigated, (i) the effects of a temperature increase by $3-4^{\circ} \mathrm{C}$ above in situ temperatures on seagrass performance; (ii) the synergistic effects of thermal stress and light reduction on seagrass traits and survival; (iii) the extent to which any potential seasonal acclimatization affects the experimental optima for growth and productivity patterns and; finally (iv) the potential impact of experimental increases in temperature on the biochemical composition and nutritional value of seagrass leaves reported in the literature in comparison with results from this study. To address these questions, $Z$. marina shoots from Ireland were exposed to different temperature treatments (ranging from 6 to $24^{\circ} \mathrm{C}$ ) combined with high $(180 \mu \mathrm{mol}$ photons $\mathrm{m}^{-2} \mathrm{~s}^{-1}$ ) versus low irradiance $(60 \mu \mathrm{mol}$ photons $\mathrm{m}^{-2} \mathrm{~s}^{-1}$ ) levels across four seasons (spring: April, summer: July, and autumn: November 2015, and winter: January 2016). We hypothesize that, (i) predicted warming will favor growth of centrally distributed $Z$. marina populations, such as Irish meadows, likely growing under temperature limitation, (ii) plants exposed to low irradiance are more vulnerable to thermal stress than plants exposed to high light, and (iii) plants incubated in different seasons display diverse thermal responses and distinct optimum of temperature for growth. Finally, (iv) we expect that warming will reduce PUFA contents and particularly omega-3, as high temperatures dismiss the requirements of unsaturation levels in photosynthetic structures.

\section{MATERIALS AND METHODS}

\section{Plant Collection}

Plants were collected from a monospecific Zostera marina L. meadow situated in western Ireland at Finavarra (FV) (53.149437N, -9.133993W), southern Galway Bay (Figure 1). This area is classified as a Special Area of Conservation (SAC), Natural Heritage Area (NHA), and described as an "unpolluted" area by the Irish Environmental Protective Area (EPA) (NPWS, 2014) (more details in Beca-Carretero et al., 2019a, 2020).

Mature apical shoots were manually harvested by SCUBA diving or snorkeling at a shallow depth of 2-3 meters at intervals of 5-10 meters along a $50 \mathrm{~m}$ transect line to prevent potential resampling of the same genotypes. At this depth range, the meadow is denser and more homogenous than at shallower or deeper areas (Beca-Carretero et al., 2019a). We selected shoots with similar weights (average of $2.7 \pm 0.6 \mathrm{~g} \mathrm{DW} \mathrm{shoot}^{-1}$ ) and size (average of $32.3 \pm 8.3 \mathrm{~cm} \mathrm{shoot}^{-1}$ ) and the number of mature rhizome segments (3-4). Shoots were transferred to cooling tanks filled with ambient seawater and directly transported to the laboratory at the National University of Ireland Galway campus (Supplementary Figure 1) within $1 \mathrm{~h}$. The collected samples 


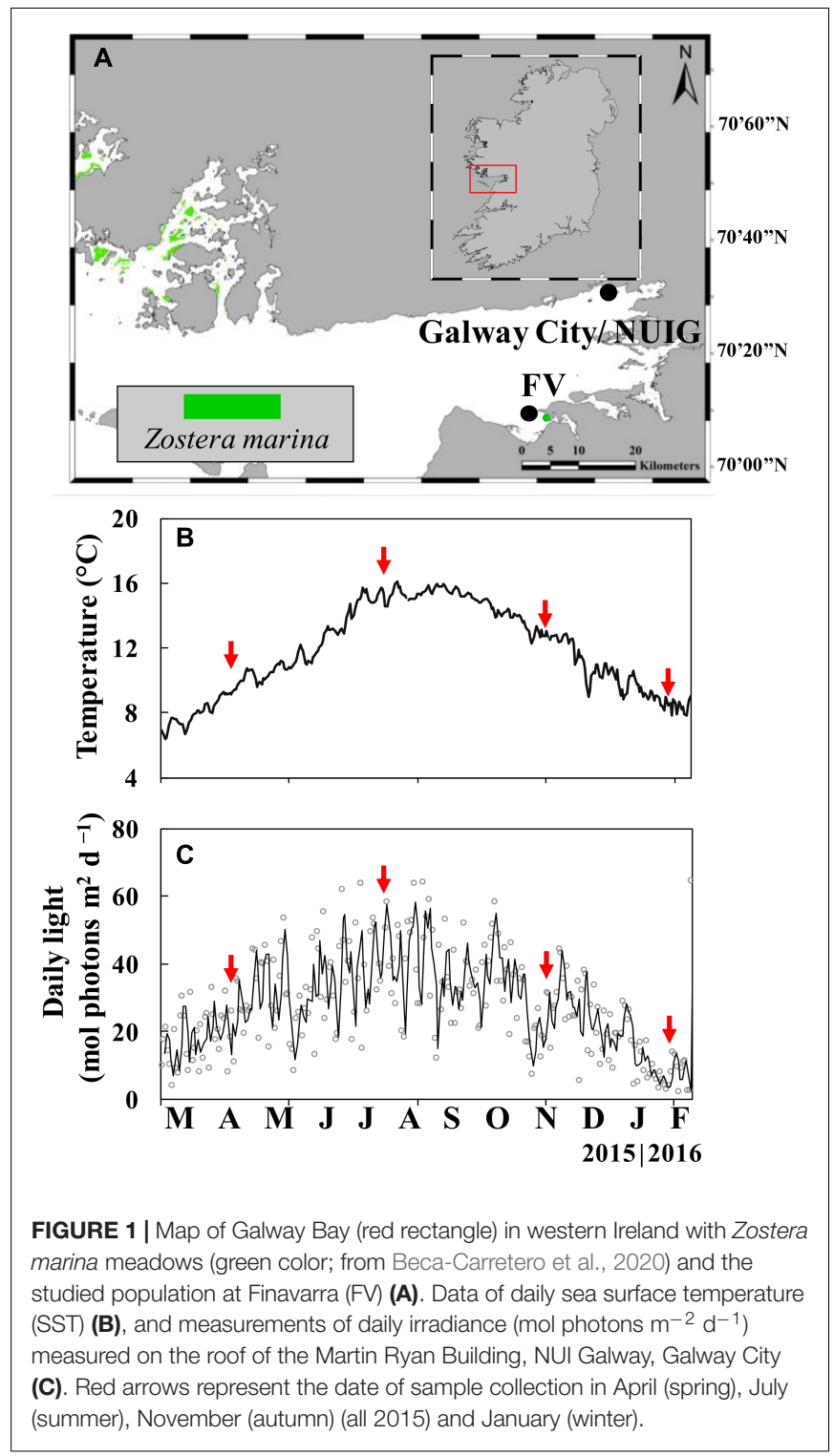

were carefully cleaned, e.g., any remnants of sediment, epiphytic organisms or dead plant materials were removed from the shoots.

\section{Details of Experimental Set-Up}

We conducted laboratory-controlled experiments on Z. marina plants across four different times of year (April, July, November 2015 and January 2016), and each time, exposed samples to combined temperature $\left(6,12,16,20\right.$ and $\left.24^{\circ} \mathrm{C}\right)$ and irradiance (180 $\mu$ mol photons $\mathrm{m}^{-2} \mathrm{~s}^{-1}$ and $60 \mu \mathrm{mol}$ photons $\mathrm{m}^{-2} \mathrm{~s}^{-1}$ ) treatments (Figure 2).

The experimental temperature range represented the natural annual range of sea surface temperature (SST) in western Ireland $\left(\sim 5-17^{\circ} \mathrm{C}\right)$ (Supplementary Figure 1); the $20^{\circ} \mathrm{C}$ treatment represented the predicted increases of $2.6-3.1^{\circ} \mathrm{C}$ of SST for the study region by the end of 2100 (IPCC, 2014; Hobday et al., 2016; Tinker and Howes, 2020); $24^{\circ} \mathrm{C}$ temperature represented an elevation by $+3-4^{\circ} \mathrm{C}$ above maximum in situ SST at the collection site ( 19-20 ${ }^{\circ} \mathrm{C}$, Stengel et al., 1999$)$ that may be reached during a summer heat-wave events in the Atlantic Ocean (Feudale and Shukla, 2011). The duration of the temperature treatment was selected to simulate the duration of previous warming episodes in temperate regions (Hobday et al., 2016).

For each temperature treatment, two irradiance levels (180 $\mu \mathrm{mol}$ photons $\mathrm{m}^{-2} \mathrm{~s}^{-1}$ and $60 \mu \mathrm{mol}$ photons $\mathrm{m}^{-2} \mathrm{~s}^{-1}$ ) were tested. Light levels were chosen based on in situ values recorded in September 2014 (maximum annual temperatures of $16-17^{\circ} \mathrm{C}$ ) during (typical) cloudy days at the location where plants were collected for the experiment. It is noteworthy that in western Ireland $63 \%$ of days have some precipitation and more than $96 \%$ days have more than $20 \%$ cloud cover ${ }^{1}$. Therefore, we considered that using light levels recorded during cloudy days were ecologically more relevant for Irish coastal ecosystems than those during sunny days. Irradiances measured using a DIVINGPAM- $\mathrm{II}^{2}$ along a $50 \mathrm{~m}$ transect long were $174.7 \pm 42.4 \mu \mathrm{mol}$ photons $\left.\mathrm{m}^{-2} \mathrm{~s}^{-1}[n=8]\right)$ at intermediate depths of Irish seagrass meadows $(2-2.5 \mathrm{~m})$, which is $\sim 70 \%$ higher than at deeper regions of the meadow (4-5 $\mathrm{m} ; 57.6 \pm 25.4 \mu \mathrm{mol}$ photons $\mathrm{m}^{-2}$ $\left.\mathrm{s}^{-1}[n=8]\right)$ (Beca-Carretero et al., 2019b). Similar light levels were also previously used in previous $Z$. marina experiments (e.g., Evans et al., 1986; Höffle et al., 2011; Staehr and Borum, 2011; Beca-Carretero et al., 2018a). A cycle of $12 \mathrm{~h}: 12 \mathrm{~h}$ light (L): dark (D) was chosen to represent an intermediate step between winter and summer daylengths (Table 1 and Supplementary Figure 1).

After collection, and prior to each experimental treatment, 120-150 plants were kept for 5 days in $20 \mathrm{~L}$ tanks at temperatures measured in situ at the time of collection (April: $11^{\circ} \mathrm{C}$; July: $17^{\circ} \mathrm{C}$; November: $11^{\circ} \mathrm{C}$; January: $6^{\circ} \mathrm{C}$ ), at a salinity of $\sim 35 \mathrm{PSU}$, and at an intermediate experimental irradiance of $120 \mu \mathrm{mol}$ photons $\mathrm{m}^{-2} \mathrm{~s}^{-1}$ in a L: D cycle of $12 \mathrm{~h}: 12 \mathrm{~h}$ (Beca-Carretero et al., 2018a; Figure 2; Table 1).

Before starting the experiment, shoots were progressively acclimated from in situ temperature to the target experimental temperature $\left(6,12,16,20\right.$, and $\left.24^{\circ} \mathrm{C}\right)$ by gradually increasing or reducing chamber temperatures by $1^{\circ} \mathrm{C}$ every $24-48 \mathrm{~h}$ (Figure 2 ). Also, plants were progressively acclimated to the target irradiance applied (60 or $180 \mu \mathrm{mol}$ photons $\mathrm{m}^{-2} \mathrm{~s}^{-1}$ ) by increasing or reducing $\sim 30 \mu \mathrm{mol}$ photons $\mathrm{m}^{-2} \mathrm{~s}^{-1}$ every 5-7 days. The experiment was started when all treatments reached their target temperatures and irradiance to ensure that all plants were pre-acclimated to lab-conditions for the same number of days (10-14 days).

For each temperature and irradiance treatment, three individual plants were incubated in each of the three individual transparent cylinders $(n=3)$ consisting of cylindrical perspex bottles (ID $10 \mathrm{~cm}$, height $35 \mathrm{~cm}$ ) with a volume of $\sim 3.5$ L. Shoots were loosely tied to a weighed-down plastic net at the bottom of each container to maintain them in a vertical orientation. Air was supplied through pumps ensuring constant mixing and $\mathrm{CO}_{2}$ supply. $80 \%$ of the seawater in the cylinders was replaced every two to 3 days.

\footnotetext{
${ }^{1}$ https://www.meteoblue.com/

${ }^{2}$ https://www.walz.com/
} 


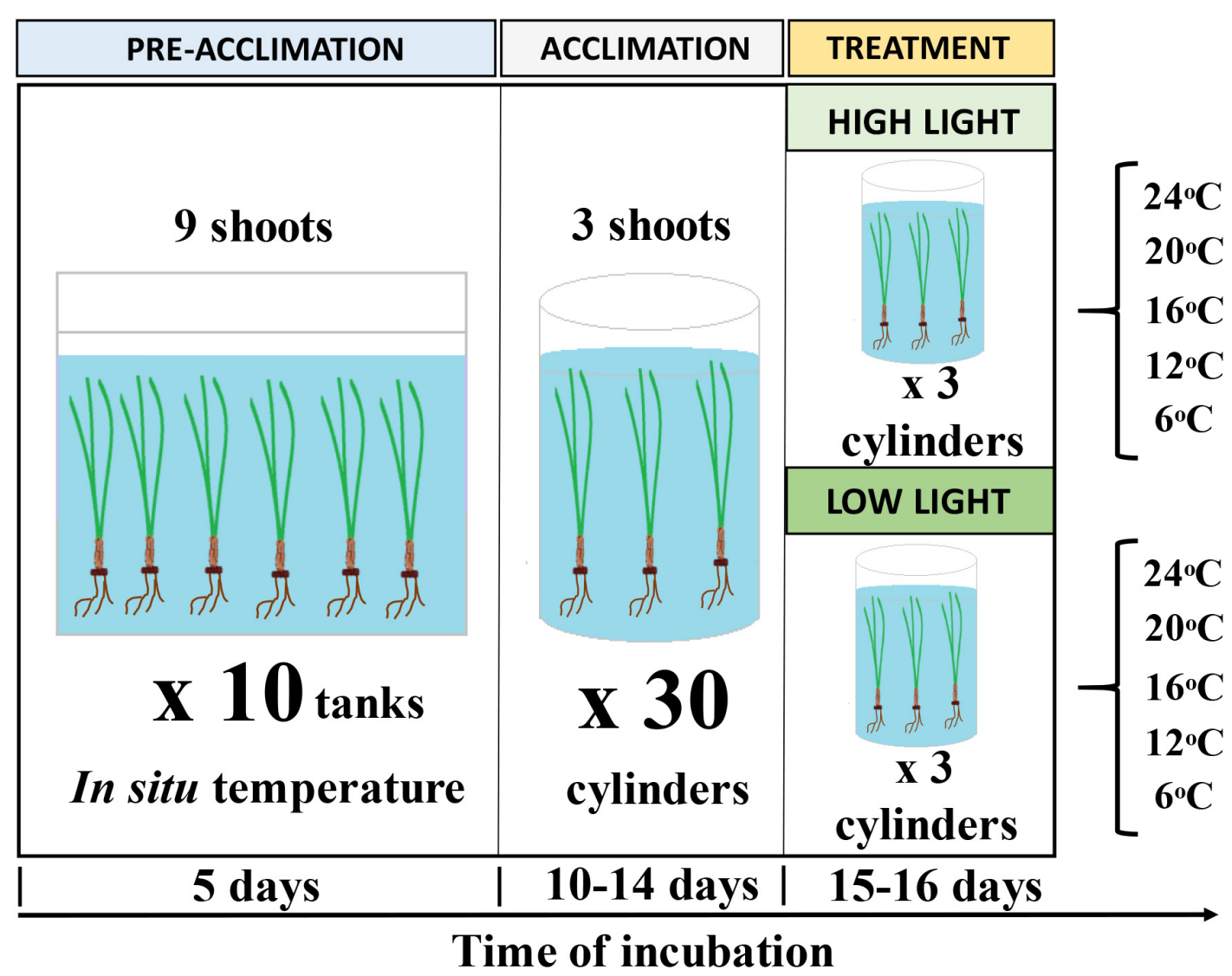

FIGURE 2 | Schematic of the experimental design. Plants of Zostera marina incubated at high (180 $\mu$ mol photons $\mathrm{m}^{-2} \mathrm{~s}^{-1}$ ) or low $60 \mu \mathrm{mol}$ photons $\left.\mathrm{m}^{-2} \mathrm{~s}^{-1}\right)$ light and 5 temperatures $\left(6,12,16,20\right.$ and $\left.24^{\circ} \mathrm{C}\right)$. Three cylinders $(n=3)$ containing three seagrass shoots were incubated in each treatment.

Irradiance levels (180 and $60 \mu \mathrm{mol}$ photons $\mathrm{m}^{-2} \mathrm{~s}^{-1}$ ) were measured inside the cylinder at the upper position of the seagrass leaves; the light was provided by Lumilux cool daylight fluorescent lamps (OSRAM L18W/865, Germany)

TABLE 1 | Geographic coordinates at Finavarra (FV), western Ireland, where Z. marina shoots were collected, water depth relative to the mean water level, in situ sea surface water temperature $(\mathrm{SST})\left({ }^{\circ} \mathrm{C}\right)$ measured at times when Z. marina biomass was collected, and averaged SST and daylength (h) (https://www.timeanddate.com) across 14 days at collection time (collection date $7 \pm$ days).

\begin{tabular}{|c|c|c|c|}
\hline Latitude & $53^{\circ} 8^{\prime} 55^{\prime \prime} \mathrm{N}$ & & \\
\hline Longitude & $-9^{\circ} 7^{\prime} 57^{\prime \prime} \mathrm{W}$ & & \\
\hline Depth (m) & $2-2.5$ & & \\
\hline & Temperature $\left({ }^{\circ} \mathrm{C}\right)$ & Temperature $\left({ }^{\circ} \mathrm{C}\right)$ & Daylength (h) \\
\hline Month & Collection time & Collection date \pm 7 days & \\
\hline Apr-15 & 11 & $9.9 \pm 0.3$ & $9.8 \pm 0.7$ \\
\hline Jul-15 & 17 & $16.1 \pm 1.1$ & $16.1 \pm 1.1$ \\
\hline Nov-15 & 12 & $11.5 \pm 0.2$ & $13.5 \pm 0.8$ \\
\hline Jan-16 & 6 & $7.7 \pm 0.1$ & $9.6 \pm 0.4$ \\
\hline
\end{tabular}

Data obtained from Beca-Carretero et al. (2019b, 2020).
(Beca-Carretero et al., 2018a). We continuously recorded water temperature using HOBO loggers (UA-002-64, Onset) installed in one cylinder per temperature and irradiance treatment. After 15-16 days, we measured a set of response parameters (see following).

\section{Morphological Descriptors}

Once the $Z$. marina shoots were acclimated to the target temperatures and before starting the experiment, all individual shoots were morphologically characterized. We measured: shoot fresh weight ( $\mathrm{g} \mathrm{FW})$, total length $\left(\mathrm{cm} \mathrm{shoot}{ }^{-1}\right)$, number of leaves $\left(\right.$ shoot $\left.^{-1}\right)$ and the length of the rhizomes $(\mathrm{cm})$. We standardized leaves and rhizome length across the all experiments to avoid potential impacts of morphology in plant responses by adjusting all shoots to two-three mature rhizome segments (2-3 cm total rhizome length) and 3-4 healthy leaves. At the end of each experimental treatment, the number of new leaves and new rhizome segments were counted, and rhizome and leaf elongation rates were assessed. To measure leaf growth (elongation) rates over time, two holes were pierced into leaves, one above the other with a distance of $2 \mathrm{~mm}$ above the basal meristem of the plant with a hypodermic needle (Sand-Jensen, 1975; Short and Duarte, 2001). We quantified the leaf formation rate (leaves shoot ${ }^{-1} \mathrm{~d}^{-1}$ ) by identifying 
the new number of leaves without punched holes, divided by the number of incubation days. Leaf elongation rate $(\mathrm{cm}$ shoot $^{-1} \mathrm{~d}^{-1}$ ) was calculated as the length of new leaf material produced during the incubation time (in days), measured (i) from the base of the meristem to the punched holes, and (ii) the length of the newly produced leaves (Short and Duarte, 2001). The relative growth rate (RGR) was calculated according to:

$$
\mathrm{RGR}=-\operatorname{Ln}\left(\frac{\mathrm{Bf}}{\mathrm{Bi}}\right) / t
$$

where $\mathrm{Bi}$ is the initial weight and Bf, the final weight of the shoot and $t$ is the incubation period in days. We haphazardly selected a group of $10 \mathrm{Z}$. marina shoots with similar size at the time of collection from the same seagrass meadow to assess the ratio of initial fresh weight (FW): dry weight (DW). Moreover, we evaluated the shoot mortality rate by the following equation:

$$
\text { Mortality rate }=-\operatorname{Ln}\left(\frac{\mathrm{Nf}}{\mathrm{Ni}}\right) / t
$$

Nf is the final shoot population ( $n=9 ; 3$ shoots per cylinder); $\mathrm{Ni}$ is the initial shoot population and $t$ is the duration of the experiment (in days).

\section{Biochemical Responses}

After 15-16 days of incubation, only healthy tissues (avoiding epiphytes or damaged parts) of the youngest and second youngest leaves were selected for biochemical analysis. Selected biomass was cleaned with distilled water prior to processing. Samples were frozen at $-20^{\circ} \mathrm{C}$ and $48 \mathrm{~h}$ later freeze-dried. The samples were then kept at $-20^{\circ} \mathrm{C}$. $24 \mathrm{~h}$ before conducting the experiments samples were again freeze-dried to remove the potential humidity.

\section{Fatty Acid Analysis}

We determined the fatty acid content and composition of $Z$. marina leaf biomass by applying the protocol previously used for macroalgae and seagrasses (Schmid et al., 2014; Beca-Carretero et al., 2018b, 2019a, 2020). Fatty acid methyl esters (FAME) were obtained by direct transmethylation of $\sim$ 20-30 mg of powdered leaf biomass with dry methanol containing $2 \%(\mathrm{v} / \mathrm{v}) \mathrm{H}_{2} \mathrm{SO}_{4}$. To prevent oxidation, vials were closed with nitrogen gas before being heated at $80^{\circ} \mathrm{C}$ for $2 \mathrm{~h}$ under continuous stirring conditions. After transmethylation, we added $1 \mathrm{~mL}$ of Milli-Q water and later extracted the FAME using $0.5 \mathrm{~mL}$ of $\mathrm{n}$-hexane. Analysis of FAME was conducted using an Agilent 7890A/5975C Gas Chromatograph/mass selective detector (GC/MSD) Series (Agilent Technologies, United States) equipped with a flame ionization detector and a fused silica capillary column (DB-WAXETR, $0.25 \mathrm{~mm} \times 30 \mathrm{~m} \times 0.25 \mu \mathrm{m}$, Agilent Technologies, Catalog No.: 122-7332). Identification of FAME was achieved by co-chromatography with authentic commercially available FAME standard of fish oil (Menhaden Oil, catalog no. 47116, Supelco). Total and individual fatty acid contents were quantified by comparison with a known quantity of added pentadecanoic acid 15:0 (99\%, catalog no. A14664-09,
Alfa Aesar, United Kingdom) as an internal standard. We added the 15:0 standard $\left(10 \mu \mathrm{l}, 5 \mathrm{mg} \mathrm{mL}^{-1}\right)$ before starting the direct transmethylation and expressed the results as the mean values of 3 replicates $(n=3)$ for each treatment.

Fatty acids (FAs) content and composition were chosen as seagrass indicator due to their proved sensitivity to environmental fluctuations including temperature and irradiance alongside its importance as nutritional compound (e.g., Falcone et al., 2004; Sanina et al., 2008; Beca-Carretero et al., 2020).

\section{Pigment Extraction}

Chlorophylls and total carotenoids were determined following two consecutive extractions, using $5 \mathrm{~mL}$ of $80 \%$ acetone each time to extract pigments from $\sim 20-30 \mathrm{mg}$ of powdered leaf biomass (Beca-Carretero et al., 2019a, 2020). The first extraction was conducted over $20 \mathrm{~h}$, and the second over $4 \mathrm{~h}$. To ensure optimal extraction and to avoid pigment oxidation, both extractions were performed in darkness at $4^{\circ} \mathrm{C}$ and with continuous stirring, and the vials were closed under nitrogen gas. After the first extraction, samples were centrifuged $(10,000 \mathrm{rpm})$ for $60 \mathrm{~s}$, the supernatant $(5 \mathrm{~mL})$ kept in darkness at $4^{\circ} \mathrm{C}$, and the remaining biomass was used for the second extraction. The supernatants of both extractions were combined to a final volume of $10 \mathrm{~mL}$, which was then used for pigment analysis $(n=3)$. We quantified chlorophyll $a, b$ and carotenoids by spectrophotometric absorbance (CARY 50 Scan UV-Visible Spectrophotometer), following the equations of Lichtenthaler and Wellburn (1983).

Chl $a\left(\mu \mathrm{g} \mathrm{mL}^{-1}\right)=12.21 \mathrm{E} 663-2.81 \mathrm{E} 646$
Chl $b\left(\mu \mathrm{g} \mathrm{mL}^{-1}\right)=20.13 \mathrm{E} 646-5.03 \mathrm{E} 663$
Carotenoids $\left(\mu \mathrm{g} \mathrm{mL} L^{-1}\right)=(1000 \mathrm{~A} 470-(3.27 \mathrm{Chl} a)-(104$
Chl $b)) / 227$
Where: Exxx $=$ Absorbance at xxx nm - Absorbance at $725 \mathrm{~nm}$

\section{Literature Review}

A thorough literature survey evaluated previous seagrass studies on biochemical components with a potential nutritional value in response to controlled temperature exposure. Any data from field experiments or in situ observations were excluded. For the literature review selected compounds include lipids, fatty acids, proteins, carbohydrates, pigments and carbon and nitrogen composition among others. We included reports on necrosis as a parameter that may affect nutritional value. Table 2 contains details of leaf compounds which varied more than $\pm 5 \%$ when seagrasses were grown under predicted warming conditions compared to in situ summer temperature. In addition, Supplementary Tables 13-14 (Excel file) include all results regardless of the degree of variation reported.

\section{Statistics}

Data of morphological descriptors, growth rates, FA content and composition and photosynthetic pigments were Ln transformed, checked for homogeneity of variance using Bartlett's test and for normality applying Kolmogorov-Smirnov 
TABLE 2 | Literature review of the effects of temperature in the nutritional composition of seagrasses leaf.

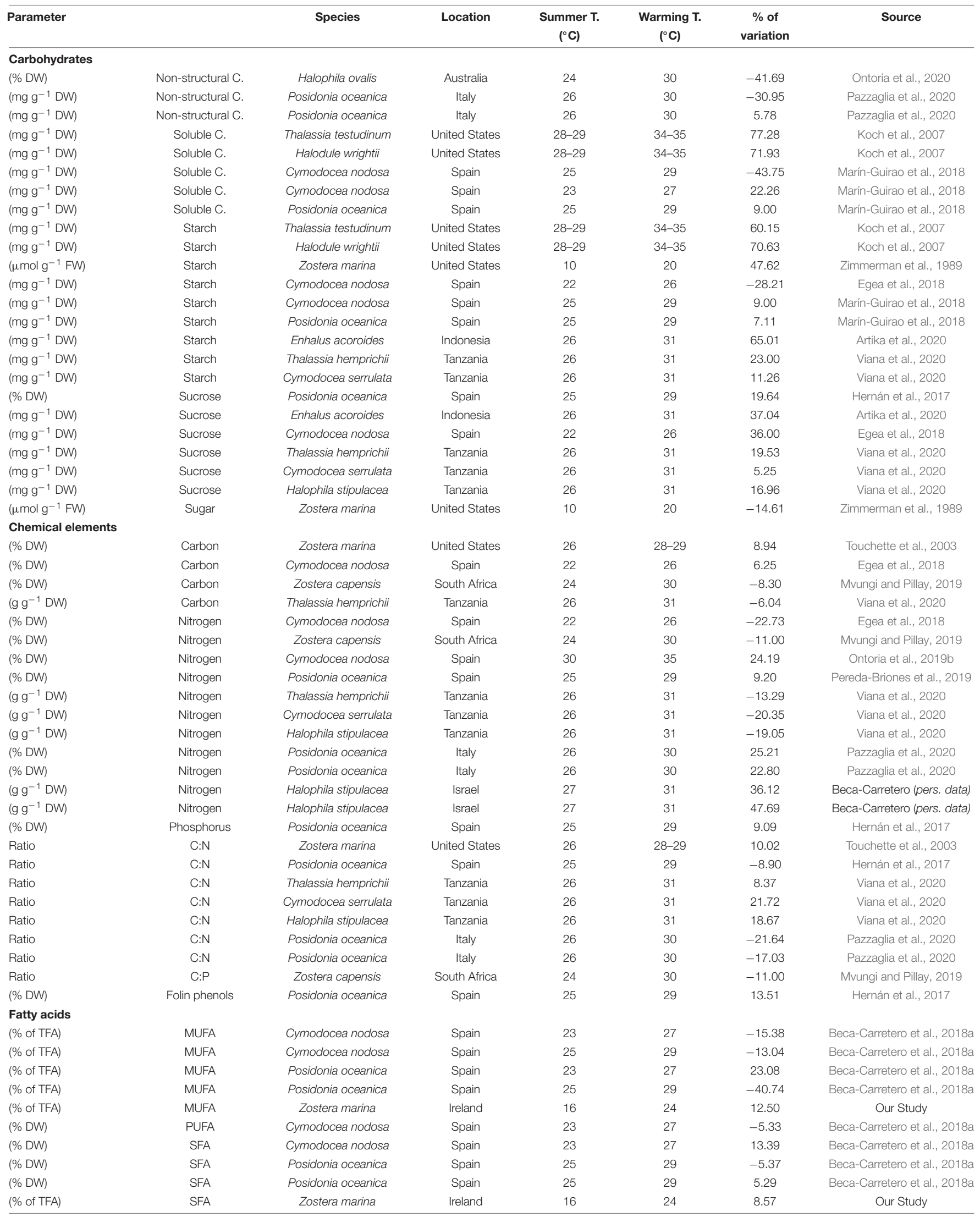


TABLE 2 | (Continued)

\begin{tabular}{|c|c|c|c|c|c|c|c|}
\hline Parameter & & Species & Location & $\begin{array}{c}\text { Summer T. } \\
\left({ }^{\circ} \mathrm{C}\right)\end{array}$ & $\begin{array}{c}\text { Warming T. } \\
\left({ }^{\circ} \mathrm{C}\right)\end{array}$ & $\begin{array}{c}\% \text { of } \\
\text { variation }\end{array}$ & Source \\
\hline$(\% \mathrm{DW})$ & TFA & Cymodocea nodosa & Spain & 25 & 29 & 7.41 & Beca-Carretero et al., 2018a \\
\hline$(\% \mathrm{DW})$ & TFA & Posidonia oceanica & Spain & 23 & 27 & 7.89 & Beca-Carretero et al., $2018 \mathrm{a}$ \\
\hline$(\% \mathrm{DW})$ & TFA & Posidonia oceanica & Spain & 25 & 29 & -5.41 & Beca-Carretero et al., 2018a \\
\hline$(\% \mathrm{DW})$ & TFA & Zostera marina & Ireland & 16 & 24 & -8.33 & Our Study \\
\hline Ratio & PUFA/SFA & Zostera marina & Ireland & 16 & 24 & -10.00 & Our Study \\
\hline (\% of TFA) & \% Omega-3 & Zostera marina & Ireland & 16 & 24 & -13.87 & Our Study \\
\hline Ratio & Omega 3/6 & Cymodocea nodosa & Spain & 23 & 27 & -15.38 & Beca-Carretero et al., 2018a \\
\hline Ratio & Omega 3/6 & Cymodocea nodosa & Spain & 25 & 29 & -13.04 & Beca-Carretero et al., $2018 a$ \\
\hline Ratio & Omega 3/6 & Posidonia oceanica & Spain & 23 & 27 & 23.08 & Beca-Carretero et al., $2018 \mathrm{a}$ \\
\hline Ratio & Omega 3/6 & Posidonia oceanica & Spain & 25 & 29 & -40.74 & Beca-Carretero et al., 2018a \\
\hline Ratio & Omega 3/6 & Zostera marina & Ireland & 16 & 24 & -28.57 & Our Study \\
\hline \multicolumn{8}{|l|}{ Proteins } \\
\hline$\left(\mathrm{mg} \mathrm{g}^{-1} \mathrm{FW}\right)$ & Protein & Halophila stipulacea & Cyprus & 26 & 32 & 42.86 & Nguyen et al., 2020a \\
\hline$\left(\mathrm{mg} \mathrm{g}^{-1} \mathrm{FW}\right)$ & Protein & Halophila stipulacea & Israel & 26 & 32 & -36.67 & Nguyen et al., 2020a \\
\hline \multicolumn{8}{|l|}{ Pigments } \\
\hline$\left(\mu \mathrm{gg}^{-1} \mathrm{FW}\right)$ & Carotenoids & Zostera noltei & Portugal & 18 & 22 & 55.93 & Repolho et al., 2017 \\
\hline$\left(\mathrm{mg} \mathrm{g}^{-1} \mathrm{DW}\right)$ & Carotenoids & Zostera marina & Ireland & 16 & 24 & -25.00 & Our Study \\
\hline$\left(\mathrm{mg} \mathrm{g}^{-1} \mathrm{FW}\right)$ & Chl. a & Zostera marina & United States & 10 & 20 & 48.48 & Zimmerman et al., 1989 \\
\hline$\left(\mathrm{mg} \mathrm{g}^{-1} \mathrm{FW}\right)$ & Chl. a & Zostera marina & China & 20 & 25 & -9.09 & Niu et al., 2012 \\
\hline$\left(\mu \mathrm{g} \mathrm{cm}^{-2}\right)$ & Chl. a & Zostera marina & China & 20 & 32 & -27.63 & Gao et al., 2017 \\
\hline$\left(\mu \mathrm{gg}^{-1} \mathrm{FW}\right)$ & Chl. a & Zostera noltei & Portugal & 18 & 22 & -35.79 & Repolho et al., 2017 \\
\hline$\left(\mu \mathrm{g} \mathrm{cm}^{-2}\right)$ & Chl. a & Halophila decipiens & Australia & 26 & 30 & 9.09 & Chartrand et al., 2018 \\
\hline$\left(\mu \mathrm{g} \mathrm{cm}^{-2}\right)$ & Chl. a & Halophila spinulosa & Australia & 26 & 30 & 27.91 & Chartrand et al., 2018 \\
\hline$\left(\mathrm{mg} \mathrm{g}^{-1} \mathrm{DW}\right)$ & Chl. a & Posidonia australis & Australia & 26 & 32 & -19.00 & Nguyen et al., 2020b \\
\hline$\left(\mathrm{mg} \mathrm{g}^{-1} \mathrm{DW}\right)$ & Chl. a & Zostera marina & Ireland & 16 & 24 & -23.53 & Our Study \\
\hline$\left(\mathrm{mg} \mathrm{g}^{-1} \mathrm{FW}\right)$ & Chl. $b$ & Zostera marina & United States & 10 & 20 & 36.59 & Zimmerman et al., 1989 \\
\hline$\left(\mathrm{mg} \mathrm{g}^{-1} \mathrm{FW}\right)$ & Chl. $b$ & Zostera marina & China & 20 & 25 & -14.29 & Niu et al., 2012 \\
\hline$\left(\mu \mathrm{g} \mathrm{g}^{-1} \mathrm{FW}\right)$ & Chl. $b$ & Zostera noltei & Portugal & 18 & 22 & -15.31 & Repolho et al., 2017 \\
\hline$\left(\mu \mathrm{g} \mathrm{cm}^{-2}\right)$ & Chl. $b$ & Halophila decipiens & Australia & 26 & 30 & 20.00 & Chartrand et al., 2018 \\
\hline$\left(\mu \mathrm{g} \mathrm{cm}^{-2}\right)$ & Chl. $b$ & Halophila spinulosa & Australia & 26 & 30 & 36.36 & Chartrand et al., 2018 \\
\hline$\left(\mathrm{mg} \mathrm{g}^{-1} \mathrm{DW}\right)$ & Chl. $b$ & Zostera marina & Ireland & 16 & 24 & -21.43 & Our Study \\
\hline$\left(\mu \mathrm{g} \mathrm{g}^{-1} \mathrm{FW}\right)$ & Phaeo. a & Zostera noltei & Portugal & 18 & 22 & 355.62 & Repolho et al., 2017 \\
\hline$\left(\mu \mathrm{g} \mathrm{g}^{-1} \mathrm{FW}\right)$ & Phaeo. $b$ & Zostera noltei & Portugal & 18 & 22 & 146.24 & Repolho et al., 2017 \\
\hline$\left(\mathrm{mg} \mathrm{g}^{-1} \mathrm{FW}\right)$ & Chl. $a+b$ & Zostera marina & United States & 10 & 20 & 43.93 & Zimmerman et al., 1989 \\
\hline$\left(\mathrm{mg} \mathrm{g}^{-1} \mathrm{FW}\right)$ & Chl. $a+b$ & Zostera marina & China & 20 & 25 & -11.88 & Niu et al., 2012 \\
\hline$\left(\mu \mathrm{g} \mathrm{g}^{-1} \mathrm{FW}\right)$ & Chl. $a+b$ & Zostera noltei & Portugal & 18 & 22 & -29.80 & Repolho et al., 2017 \\
\hline$\left(\mathrm{mg} \mathrm{g}^{-1} \mathrm{DW}\right)$ & Chl. $a+b$ & Zostera marina & Greenland & 20 & 28 & 13.51 & Beca-Carretero et al., 2018b \\
\hline$\left(\mu \mathrm{g} \mathrm{cm}^{-2}\right)$ & Chl. $a+b$ & Halophila decipiens & Australia & 26 & 30 & 15.09 & Chartrand et al., 2018 \\
\hline$\left(\mu \mathrm{g} \mathrm{cm}^{-2}\right)$ & Chl. $a+b$ & Halophila spinulosa & Australia & 26 & 30 & 34.38 & Chartrand et al., 2018 \\
\hline$\left(\mathrm{mg} \mathrm{g}^{-1} \mathrm{DW}\right)$ & Chl. $a+b$ & Halophila stipulacea & Israel & 26 & 32 & -45.45 & Nguyen et al., 2020a \\
\hline$\left(\mathrm{mg} \mathrm{g}^{-1} \mathrm{DW}\right)$ & Chl. $a+b$ & Halophila stipulacea & Cyprus & 26 & 32 & 38.24 & Nguyen et al., 2020a \\
\hline$\left(\mathrm{mg} \mathrm{g}^{-1} \mathrm{DW}\right)$ & Chl. $a+b$ & Zostera marina & Ireland & 16 & 24 & -20.83 & Our Study \\
\hline Ratio & Chl. $a / b$ & Zostera marina & China & 20 & 32 & -7.50 & Gao et al., 2017 \\
\hline Ratio & Chl. $a / b$ & Halophila decipiens & Australia & 26 & 30 & -9.52 & Chartrand et al., 2018 \\
\hline Ratio & Chl. $a / b$ & Halophila spinulosa & Australia & 26 & 30 & -8.59 & Chartrand et al., 2018 \\
\hline Ratio & Chl. $a / b$ & Zostera marina & China & 20 & 25 & 5.26 & Niu et al., 2012 \\
\hline Ratio & Chl. b/a & Posidonia australis & Australia & 26 & 32 & 7.00 & Nguyen et al., 2020b \\
\hline Ratio & Carot.:Chl. & Zostera noltei & Portugal & 18 & 22 & 88.46 & Repolho et al., 2017 \\
\hline$\left(\mathrm{mg} \mathrm{g}^{-1} \mathrm{DW}\right)$ & Total content & Zostera marina & Ireland & 16 & 24 & -20.00 & Our Study \\
\hline$\left(\mu \mathrm{g} \mathrm{cm}^{-2}\right)$ & Total content & Posidonia oceanica & Italy & 26 & 30 & 13.14 & Pazzaglia et al., 2020 \\
\hline$\left(\mu g g^{-1} \mathrm{FW}\right)$ & Auroxanthin & Zostera noltei & Portugal & 18 & 22 & 159.99 & Repolho et al., 2017 \\
\hline$\left(\mu \mathrm{g} \mathrm{g}^{-1} \mathrm{FW}\right)$ & Antheraxanthin & Zostera noltei & Portugal & 18 & 22 & 109.77 & Repolho et al., 2017 \\
\hline$(\% \mathrm{DW})$ & Violaxanthin & Zostera muelleri & Australia & 27 & 30 & -13.41 & York et al., 2013 \\
\hline$\left(\mu \mathrm{g} \mathrm{g}^{-1} \mathrm{FW}\right)$ & Violaxanthin & Zostera noltei & Portugal & 18 & 22 & -16.71 & Repolho et al., 2017 \\
\hline$\left(\mathrm{mg} \mathrm{mm}^{-2}\right)$ & Zeaxanthin & Zostera muelleri & Australia & 27 & 30 & 102.40 & York et al., 2013 \\
\hline$\left(\mu \mathrm{g} \mathrm{g}^{-1} \mathrm{FW}\right)$ & Zeaxanthin & Zostera noltei & Portugal & 18 & 22 & 24.34 & Repolho et al., 2017 \\
\hline$\left(\mu \mathrm{g} \mathrm{g}^{-1} \mathrm{FW}\right)$ & $\beta$-carotene & Zostera noltei & Portugal & 18 & 22 & 44.79 & Repolho et al., 2017 \\
\hline$\left(\mu \mathrm{g} \mathrm{g}^{-1} \mathrm{FW}\right)$ & Luteín & Zostera noltei & Portugal & 18 & 22 & 42.33 & Repolho et al., 2017 \\
\hline \multicolumn{8}{|c|}{ Other indicators } \\
\hline Ratio & De-Epoxidation & Zostera muelleri & Australia & 27 & 30 & 70.00 & York et al., 2013 \\
\hline Ratio & De-Epoxidation & Zostera noltei & Portugal & 18 & 22 & 10.26 & Repolho et al., 2017 \\
\hline
\end{tabular}


TABLE 2 | (Continued)

\begin{tabular}{|c|c|c|c|c|c|c|c|}
\hline Parameter & & Species & Location & $\begin{array}{c}\text { Summer T. } \\
\left({ }^{\circ} \mathrm{C}\right)\end{array}$ & $\begin{array}{c}\text { Warming T. } \\
\left({ }^{\circ} \mathrm{C}\right)\end{array}$ & $\begin{array}{c}\% \text { of } \\
\text { variation }\end{array}$ & Source \\
\hline \multicolumn{8}{|l|}{ Necrosis } \\
\hline (leaf surface \%) & Necrosis & Cymodocea rotundata & Australia & 22.7 & 43 & 69.74 & Collier and Waycott, 2014 \\
\hline (leaf surface \%) & Necrosis & Halodule uninervis & Australia & 22.7 & 43 & 89.74 & Collier and Waycott, 2014 \\
\hline (leaf surface \%) & Necrosis & Thalassia hemprichii & Australia & 22.7 & 43 & 94.36 & Collier and Waycott, 2014 \\
\hline (leaf surface \%) & Necrosis & Posidonia oceanica & Spain & 20 & 32 & 59.30 & Traboni et al., 2018 \\
\hline (leaf surface \%) & Necrosis & Posidonia oceanica & Spain & 20 & 35 & 9.32 & Ontoria et al., 2019a \\
\hline (leaf surface \%) & Necrosis & Cymodocea nodosa & Spain & 30 & 35 & 16.94 & Ontoria et al., 2019b \\
\hline (leaf surface \%) & Necrosis & Cymodocea nodosa & Spain & 30 & 35 & 32.37 & Ontoria et al., 2019b \\
\hline$\left(\mathrm{cm}^{2}\right)$ & Necrosis & Posidonia oceanica & Spain & 25 & 29 & 575.71 & Pereda-Briones et al., 2019 \\
\hline (leaf surface \%) & Necrosis & Posidonia oceanica & Italy & 26 & 30 & 13.20 & Pazzaglia et al., 2020 \\
\hline
\end{tabular}

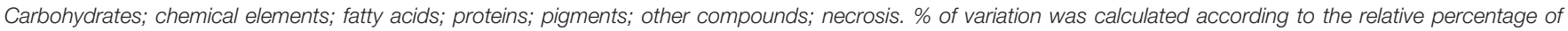

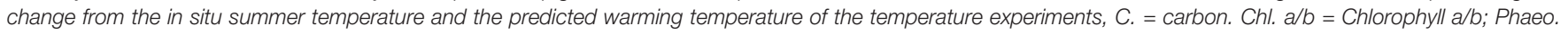
$a / b=$ Phaeophytin $a / b$.

test. As data did not meet the criteria, the non-parametric test PERMANOVA analyses based on a similarity matrix created from the Euclidean distances were implemented. We performed two PERMANOVA models. The first model aimed at investigating differences in seagrass descriptors responses to month (April, July, November 2015 and January 2016), temperature $\left(6,12,16,20\right.$, and $\left.24^{\circ} \mathrm{C}\right)$ and irradiance levels $\left(180 \mu \mathrm{mol}\right.$ photons $\mathrm{m}^{-2} \mathrm{~s}^{-1}$ vs. $60 \mu \mathrm{mol}$ photons $\left.\mathrm{m}^{-2} \mathrm{~s}^{-1}\right)$. We used "temperature," "irradiance," and "month" as fixed factors. A pairwise test was applied to identify the treatments that differed significantly $(p<0.05)$. The second model was implemented to assess responses of seagrass traits to temperature and irradiance treatments each separate month. In this model "temperature" and "irradiance" were fixed factors. A pairwise test was applied to identify the treatments that differed significantly $(p<0.05)$. All data treatments and statistical analysis were performed using the PRIMERandPERMANOVA 6 statistical package. Growth rates, FAs and pigment concentrations are reported as means and standard deviation (SD).

Pearson correlation analysis was used to assess potential relationships between FA composition (e.g., PUFA, SFA) and experimental temperatures $\left(6,12,16,20\right.$, and $\left.24^{\circ} \mathrm{C}\right)$ including both irradiance levels $\left(180 \mu \mathrm{mol}\right.$ photons $\mathrm{m}^{-2} \mathrm{~s}^{-1}$ vs. $60 \mu \mathrm{mol}$ photons $\mathrm{m}^{-2} \mathrm{~s}^{-1}$ ).

\section{RESULTS}

\section{Effect of Temperature on Plant Traits}

Temperature treatment significantly affected growth of $Z$. marina plants in terms of leaf elongation, leaf formation, rhizome elongation, internode formation and RGR (PERMANOVA, $p<0.01)$. Overall, most growth descriptors displayed bellshaped patterns, with $16-20^{\circ} \mathrm{C}$ representing more favorable temperatures, and lowest rates reached at coldest $\left(6^{\circ} \mathrm{C}\right)$ and warmest temperatures $\left(24^{\circ} \mathrm{C}\right)$ at both irradiance levels (Figure 3 and Supplementary Tables 1-5).

Most biochemical descriptors in $Z$. marina leaves including fatty acid content and composition and photosynthetic pigments were significantly affected by temperature treatments (Figure 4 and Supplementary Table 1). The average total fatty acid (TFA) content of $Z$. marina leaves was $1.41 \pm 0.32 \%$ of $\mathrm{DW}$, however, no clear pattern in response to temperature increase was observed. The most abundant FAs were polyunsaturated fatty acids (PUFAs), which accounted for an average of $57.45 \pm 6.6 \%$ of TFA across all seasons (April, July, November and January). The most abundant PUFA was $\alpha$-linolenic acid (ALA, 18:3n-3), followed by linoleic acid (LA, 18:2 n-6) and hexadecatrienoic acid (HTA, 16:3n-3). The second most abundant group of FAs were saturated fatty acids (SFAs) which accounted for an average value of $35.1 \pm 6.6 \%$ of TFA, where palmitic acid (16:0) was the most common SFA, followed by stearic acid (18:0). Finally, monounsaturated fatty acids (MUFAs) accounted for an average of $4.3 \pm 1.5 \%$ of TFA (Supplementary Tables 6-9).

Common to all seasonal experiments, the observed reductions in PUFAs following high temperature exposure were mostly attributed to changes in $n-3$ PUFAs (18:3n-3 and 16:3n-3), and occurred at both high and low light; lowest percentages were detected at $24^{\circ} \mathrm{C}$ (Figure 5). For example, in plants incubated at higher irradiances, a significant reduction (Pearson correlation, $p<0.05, n=30$ ) of $n-3$ PUFAs from 6 to $24^{\circ} \mathrm{C}$ was observed with a decrease of $0.9 \%$ by $1^{\circ} \mathrm{C}$ increase. On the other hand, a significant increase in SFA and $n-6$ PUFA was observed with temperature (Figure 5).

\section{Effect of Low Light on Plant Traits}

Leaf and rhizome growth rates of plants in low light was significantly reduced in comparison to plants exposed to high light (PERMANOVA, $p<0.01$ ) (Figure 3 and Supplementary Table 2). For instance, plants acclimated at light-limited conditions produced new leaves and rhizome segments 21.1 and $18.7 \%$ slower than plant acclimated to high irradiances (PERMANOVA, $p<0.05$ ). At temperatures above the optimum range $\left(16-20^{\circ} \mathrm{C}\right)$, light limitation induced a significant, abrupt decline in both leaf and rhizome growth compared to plants grown at high light. Additionally, at high temperatures, mortality rates were higher in plants exposed to low light than in plants incubated at high light; at low light, $30 \%$ of the incubated shoots died when grown at $24^{\circ} \mathrm{C}$ (Supplementary Table 12). 


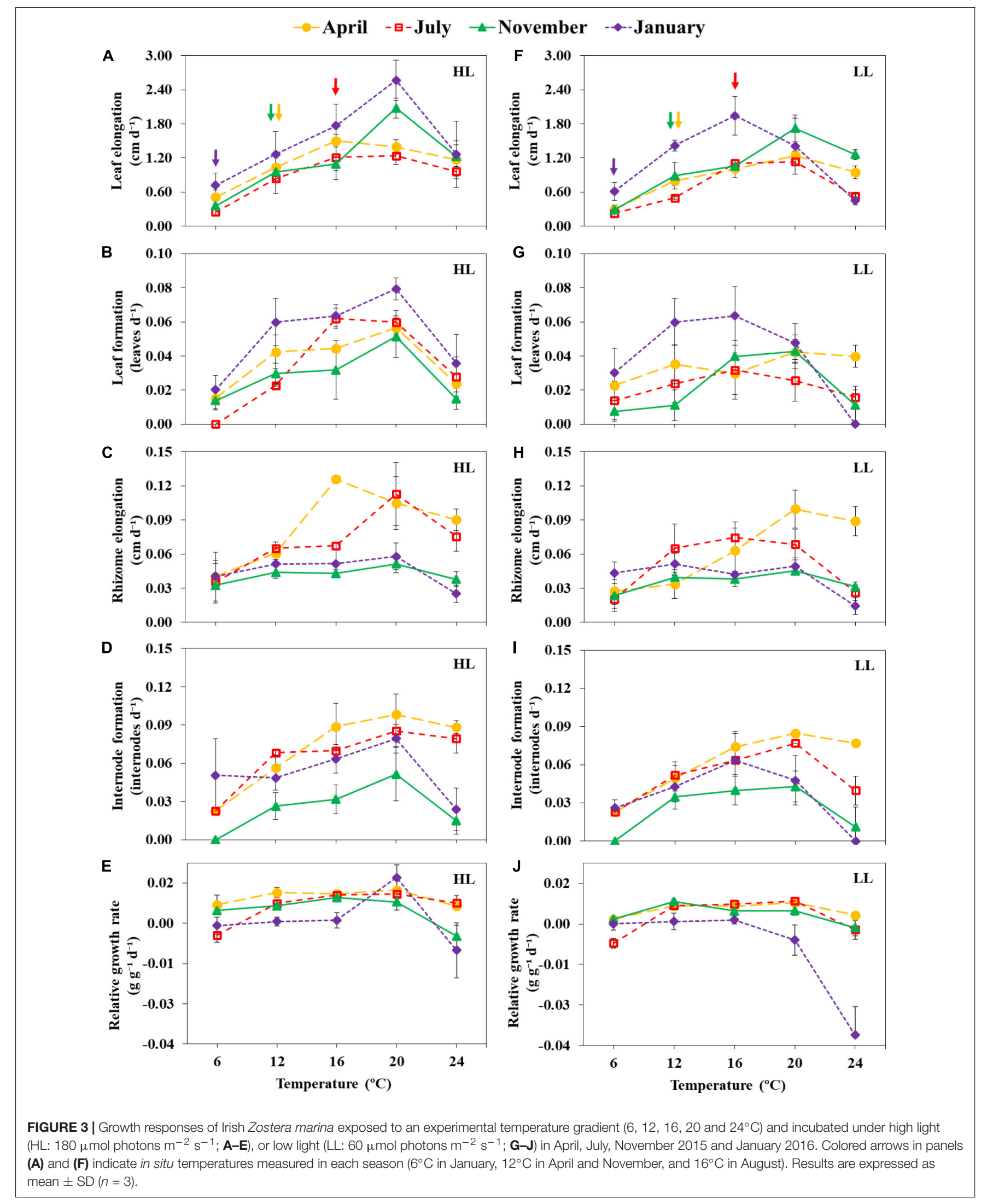



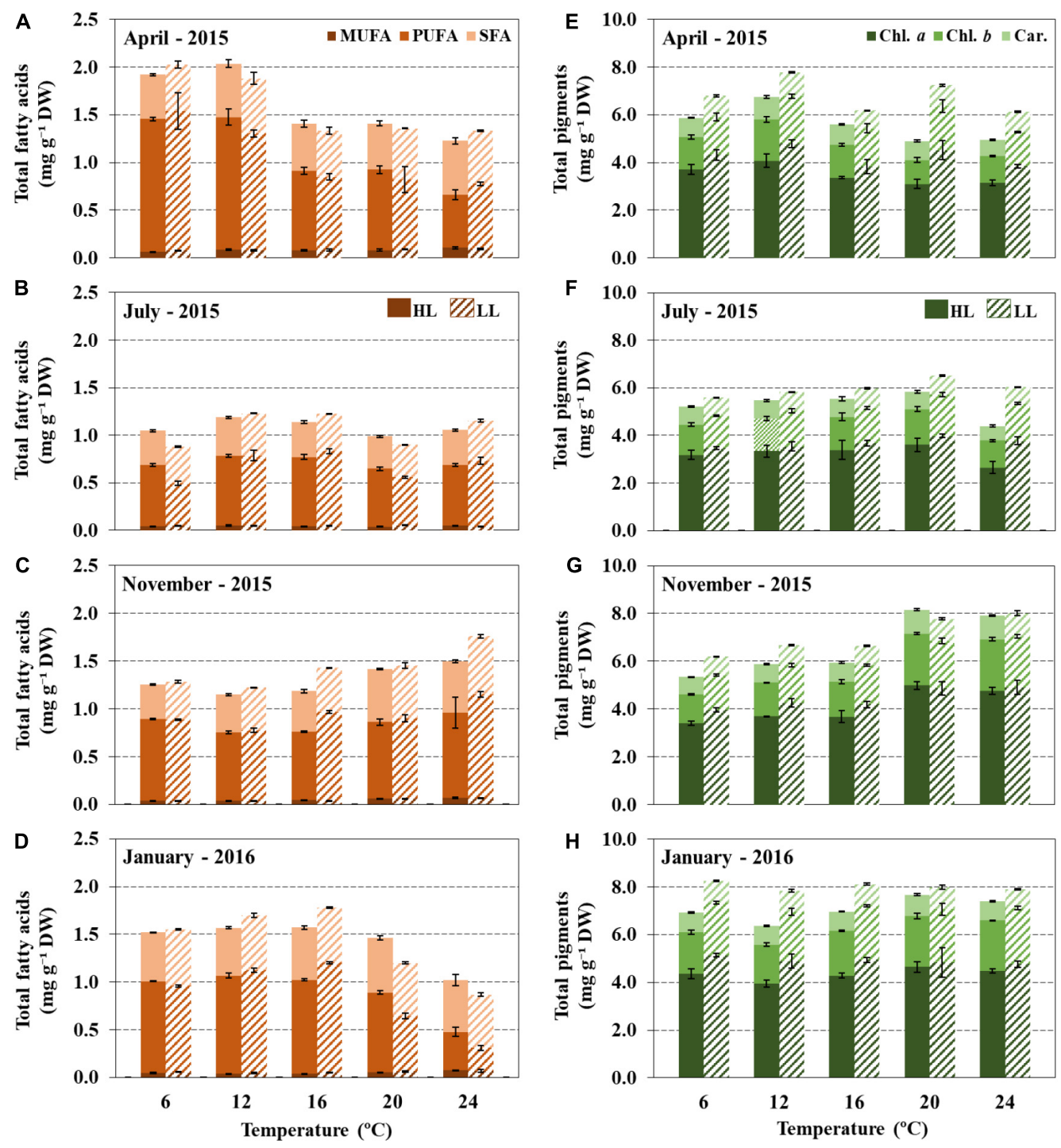

FIGURE 4 | Total fatty acid content (\% DW) and composition (\% of TFA) (A-D), and pigment composition (E-H) of leaves of Irish Zostera marina exposed to an experimental temperature gradient $\left(6,12,16,20\right.$, and $\left.24^{\circ} \mathrm{C}\right)$ and under high light ( $\mathrm{HL}, 180 \mu \mathrm{mol}$ photons $\left.\mathrm{m}^{-2} \mathrm{~s}^{-1}\right)$, or low light $\left(\mathrm{LL}, 60 \mu \mathrm{mol}\right.$ photons $\left.\mathrm{m}^{-2} \mathrm{~s}^{-1}\right)$ in April, July, November 2015 and January 2016. Results are expressed as mean \pm SD $(n=3)$.

Reduction in irradiance at optimal temperatures for growth $\left(16-20^{\circ} \mathrm{C}\right)$ generated an increase in FA unsaturation levels in leaves. The $n-3 / n-6$ PUFA ratio in $Z$. marina exposed to low light significantly increased by $9.4 \%$ in comparison to plants exposed to high light (PERMANOVA, $p<0.05$ ) (Figure 4 and Supplementary Table 2). Concentrations of photosynthetic pigments were also higher in low-light than in high lightacclimated plants (Figure 4 and Supplementary Table 10).

\section{Effect of Seasonal Acclimation on Plant Responses to Temperature and Irradiance Treatments}

At all seasons, temperature increases of $4^{\circ} \mathrm{C}$ or more above in situ temperatures significantly enhanced growth of both rhizomes and leaf structures. For instance, in the experiment conducted in April, leaf growth at $16^{\circ} \mathrm{C}$ was $44.9 \%$ higher than in plants exposed to the in situ temperature of $12^{\circ} \mathrm{C}$ (PERMANOVA, $p<0.05$, Figure 3). In summer, rhizome segments of plants incubated at $20^{\circ} \mathrm{C}$ grew $58 \%$ faster than those of plants incubated at in situ temperatures $\left(16^{\circ} \mathrm{C}\right)$. Similar trends were observed in experiments conducted in January and November (PERMANOVA, $p<0.05$, Supplementary Table 2).

Leaf and rhizome descriptors displayed different growth patterns depending on the seasonal acclimatization of the plants. Significantly higher leaf elongation and leaf formation rates were observed when the experiment was conducted in the coldest month, January (in situ temperatures of 5$6^{\circ} \mathrm{C}$ ), compared to April or July (PERMANOVA, $p<0.05$, Figure 3 and Supplementary Table 2). By contrast, significantly 

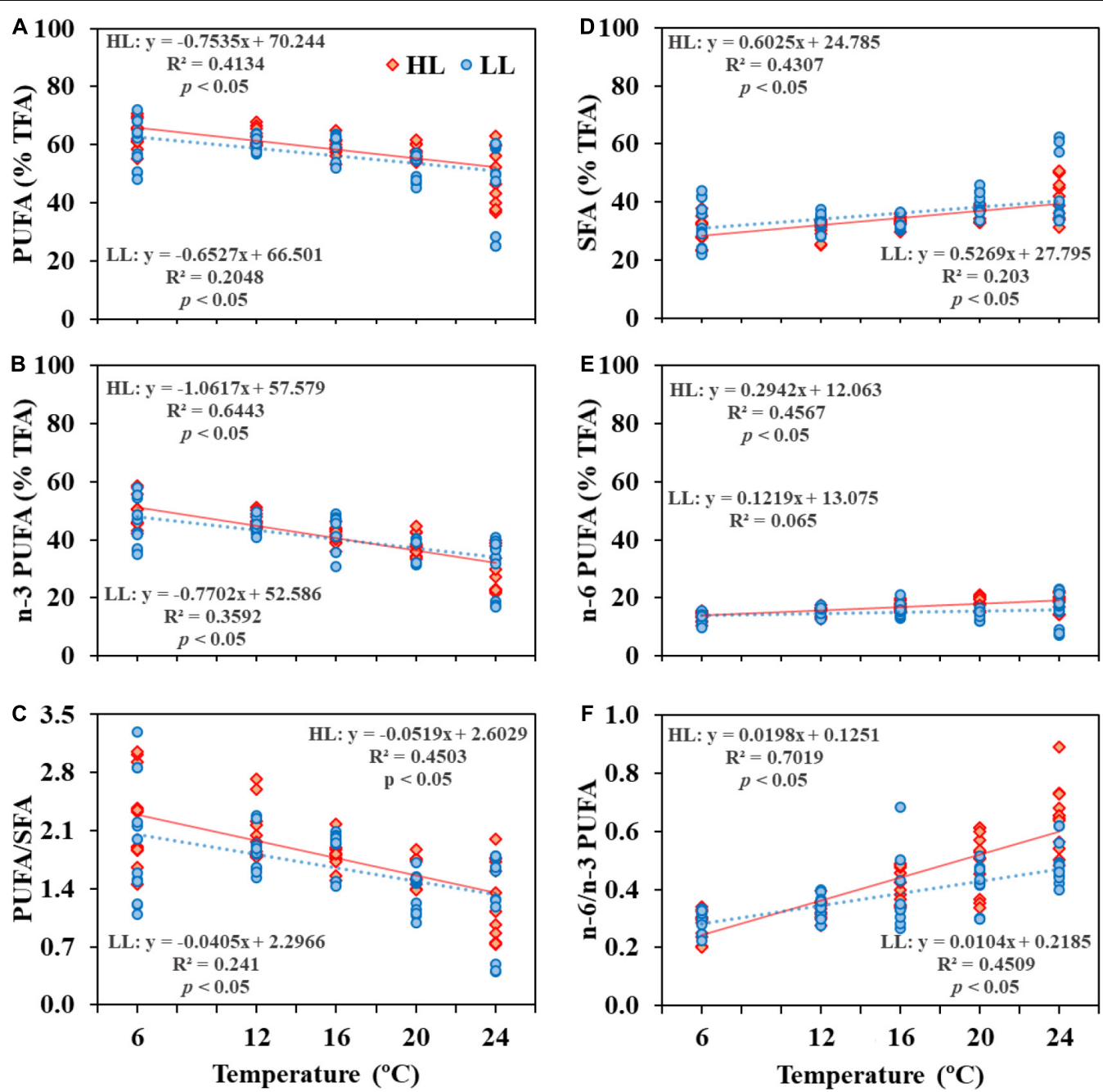

FIGURE 5 | PUFAVSFA, $n-3$ PUFA (\% of TFA), $n-3 / n-6$ PUFA ( $Y$-exe) of leaves of Irish Zostera marina versus experimental temperature $\left(6,12,16,20\right.$, and $\left.24{ }^{\circ} \mathrm{C}\right)$ (X-exe). Plants incubated under high light ( $\mathrm{HL}, 180 \mu \mathrm{mol}$ photons $\left.\mathrm{m}^{-2} \mathrm{~s}^{-1}\right)$ (A-C), or low light (LL, $\left.60 \mu \mathrm{mol}^{\mathrm{photons}} \mathrm{m}^{-2} \mathrm{~s}^{-1}\right)$ (D-F). Dashed lines represent the linear regression line $(n=36)$.

higher rhizome elongation and internode formation rates were observed in plants incubated in warmer months (April and July) than in plants collected in January and November. For instance, at optimal temperatures for growth $\left(16-20^{\circ} \mathrm{C}\right)$, rhizome elongation of plants incubated in July $(0.9 \pm 0.01 \mathrm{~cm}$ $\mathrm{d}^{-1}$ ) was 71 and $99 \%$ higher than in plants incubated in January and November, respectively. Noticeably, both leaf and rhizome growth descriptors were significantly lower in November in comparison with plants incubated during other months (PERMANOVA, $p<0.05$ ). At $24^{\circ} \mathrm{C}$, plants incubated in November and January displayed negative RGRs, but RGRs of plants incubated in April and July were positive. Mortality occurred in experiments conducted in April, November and January at $24^{\circ} \mathrm{C}$ but all plants survived in all experimental treatments in July (Supplementary Table 11), and was more accentuated in plants incubated at low light in January (HL: $0.039 \pm 0.02$ dead shoot $\mathrm{d}^{-1}$; LL: $0.054 \pm 0.02$ dead shoot $\mathrm{d}^{-1}$ ). Interestingly, $22 \%$ of the plants incubated in January at $12^{\circ} \mathrm{C}$ displayed the first stages of reproductive structures with the development of leaf sheaths corresponding to reproductive shoots (Figure 6). There was no evidence of reproductive structures in any other seasonal experiment.

TFA and photosynthetic pigment contents of $Z$. marina leaves differed significantly across seasons, however, there was no apparent clear pattern in response to temperature treatment (Figure 4) (PERMANOVA, $p<0.05$ ). For instance, in plants incubated in April, the highest TFA contents were observed at lowest temperatures $\left(6\right.$ and $12^{\circ} \mathrm{C}$ ) with average values of $2.05 \pm 0.1 \%$ of DW, and lowest contents at highest experimental temperatures $\left(24^{\circ} \mathrm{C}, 1.3 \pm 0.1 \%\right.$ of DW) (PERMANOVA, $p<0.05)$. While in November, a contrary pattern with the largest TFA contents observed at $24^{\circ} \mathrm{C}(1.7 \pm 0.2 \%$ of DW $)$ and lowest TFA were detected at lowest temperatures $\left(12^{\circ} \mathrm{C}, 1.3 \pm 0.05 \%\right.$ of DW) (Supplementary Table 10). 


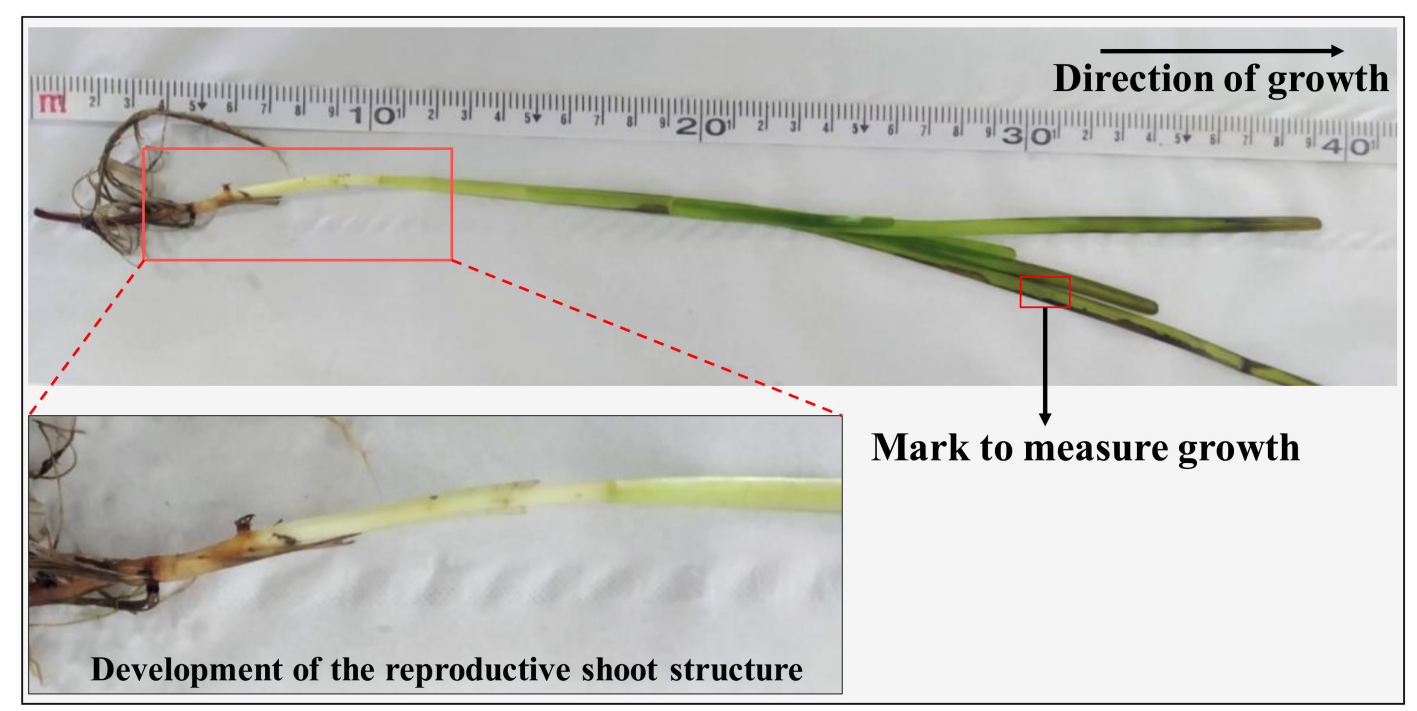

FIGURE 6 | Plant of Zostera marina incubated in January in high light (180 $\mu$ mol photons $\left.\mathrm{m}^{-2} \mathrm{~s}^{-1}\right)$ and $12^{\circ} \mathrm{C}$ showing first stages of reproductive structures. The direction of growth and growth marks are indicated in the picture.

Nearly all seasonal results revealed significant interactive effects between temperature, irradiance and time on growth descriptors (Supplementary Table 2) (PERMANOVA, $p<0.05$ ). Particularly, growth responses to temperature treatments were differently modulated by light, depending on season. For example, in January, as temperature increased, low light significantly reduced RGR in comparison to high light; on the contrary, plants incubated in April and July where not affected by light treatment (PERMANOVA, $p<0.05$ ). Additionally, rhizome elongation of plants incubated in April did not display negative responses to warming $\left(20-24^{\circ} \mathrm{C}\right)$ at either light treatment (Figure 3). However, a different pattern was observed in July, with low light significantly reducing rhizome elongation.

\section{DISCUSSION}

To the best of our knowledge, this is the first study attempting to assess the potential impacts of seasonality on outcomes of controlled exposure experiments using seagrasses. Our results indicate that seasonal acclimation significantly conditioned growth and biochemical responses of seagrass to thermal and low light stress. Besides, the reported outcomes pointed out that warming will likely favor growth and production of centrally distributed Z. marina populations which represent the broader habitat range of this species. These outcomes are of high relevance in predicting the effects of climate change on seagrasses and highlight the importance of seasonal conditioning on seagrass responses.

\section{Effects of Temperature Increases on Growth Performance}

Results confirm our hypothesis that predicted warming episodes of $\sim 3-4^{\circ} \mathrm{C}$ above in situ current conditions
(IPCC, 2014), including colder periods, in temperate regions may stimulate annual increases in growth of seagrass populations currently living under temperature-limited conditions (e.g., Krause-Jensen and Duarte, 2014).

In this study, regardless of light treatment, experimental optimum growth temperatures (between 16 and $20^{\circ} \mathrm{C}$ ) for Z. marina from western Ireland were lower than previously reported for other centrally located populations of the species (Lee et al., 2007; review in Beca-Carretero et al., 2018a). Such different latitudinal responses may be explained by the local adaptations of seagrass thermal tolerance to in situ temperature conditions (Short and Neckles, 1999; Bennett et al., 2015). In the context of climate mitigation potential of seagrasses in temperate regions, the expected increases in productivity can be interpreted as a likely higher capacity to uptake and store carbon in the sediments, therefore reinforcing their role as a carbon sink (Macreadie et al., 2019). Negative effects of thermal stress were observed in most of the seagrass descriptors at $24^{\circ} \mathrm{C}$. These results are in accordance with previous studies which correlated increases in respiration, growth inhibition and shoot death with anomalous high temperatures ranging from 20 to $30^{\circ} \mathrm{C}$ (e.g., review in Lee et al., 2007; Collier and Waycott, 2014; Gao et al., 2017).

The observed warming stimulation in seagrass productivity under lower irradiances can favor their capacity to colonize deeper areas (more than 4-6 $\mathrm{m}$ in Irish coasts), as warming enhances photosynthetic efficiency of temperaturelimited seagrass populations (Krause-Jensen et al., 2012; Krause-Jensen and Duarte, 2014). In agreement with this, warm-adapted Atlantic, Pacific and Mediterranean Z. marina populations can colonize depths of 10-20 m (Morita et al., 2007; Ondiviela et al., 2014; Boutahar et al., 2020), while the vertical distribution of central and northern $Z$. marina 
populations is restricted to 4-10 $\mathrm{m}$ (e.g., Phillips, 1974; Krause-Jensen et al., 2011; Beca-Carretero et al., 2020). It is noteworthy that other factors such as greater light penetration can also explain the colonization of deeper areas within its southern distribution range such as in Mediterranean regions. In addition, both marine and freshwater macrophytes were reported to extend their vertical distribution to greater depths in response to increases in temperature (Duarte and Kalff, 1987; Rooney and Kalff, 2000; Jorda et al., 2020). As expected, plants growing at limiting irradiances had lower biomass production in both above and belowground tissues, probably explained by lower photosynthetic activity and carbon acquisition (e.g., Lee and Dunton, 1997; Ruiz and Romero, 2003).

\section{Synergistic Effects of Thermal Stress and Light Reduction on Seagrass Traits and Survival}

Results confirm that plants living at reduced irradiances or are experiencing episodes of high turbidity in the water column may be more vulnerable to warming episodes (e.g., Longstaff and Dennison, 1999; Krause-Jensen et al., 2005). In our study, at higher temperatures, growth was reduced at low light compared to high light, and there were more visible signs of leaf necrosis alongside higher mortality rates in most seasonal experiments. A recent study reported largescale declines of $Z$. marina populations after a combined effect of light reduction due to precipitation events alongside anomalous warming episodes (Johnson et al., 2021). The authors also found a rapid demographic recovery, mostly explained by the high abundance of the seedling. Studies in the Mediterranean Sea show that extreme summer temperatures provoked high mortality in deep-adapted (17-25 m) populations of Posidonia oceanica which were not able to recover in subsequent years, whereas shallow populations were less affected and recovered fully (Marbà and Duarte, 2010; Aoki et al., 2020). Additionally, projected sea-level rise will further reduce the irradiance available to $Z$. noltei plants distributed at their deeper range $(\sim 2-2.8 \mathrm{~m})$, resulting in more extreme conditions and physiological stress (Ondiviela et al., 2020).

Interestingly, proportions of $n-3$ PUFA relative to $n-6$ PUFA in plants receiving less light increased significantly, thus increasing the level of unsaturation of FA in leaves. Similar results were previously observed in the tropical seagrass $H$. stipulacea (Beca-Carretero et al., 2019a) and also in other marine primary producers (Guihéneuf and Stengel, 2013). This regulation of FA unsaturation levels in photosynthetic structures under light limitation may represent a photoadaptative mechanism enabling optimal photosynthetic and physiological performance (Gombos et al., 1994; Sanina et al., 2008; Solovchenko et al., 2008). Regarding pigmentation, plants exposed to low light accumulated higher contents of chlorophyll than those exposed to higher irradiances. This is a well-described mechanism of seagrasses to favor photosynthetic efficiency in low-light environments (e.g., Olesen et al., 2002; York et al., 2013).

\section{Effects of Seasonal Modulation on Seagrass Responses to Temperature Increases and Irradiance Reduction}

Experimental growth rates measured across seasons at respective in situ temperatures $\left(6^{\circ} \mathrm{C}\right.$ in January, $12^{\circ} \mathrm{C}$ in April and November and $16^{\circ} \mathrm{C}$ in August) were highly similar to those measured in the field in shallow seagrass meadows in western Ireland $\left(53.3272^{\circ} \mathrm{N}, 9.6168^{\circ} \mathrm{W}\right)$ (Supplementary Table 12). These results confirm the robustness of our experimental conditions and resultant response measurements but also highlight the importance of capturing seasonal responses of temperate seagrasses to in situ conditions.

Unexpectedly, optimal conditions for growth $\left(16-20^{\circ} \mathrm{C}\right)$ were consistent across all seasons but critically, seasonality clearly modulated growth patterns of above and belowground compartments. On the one hand, higher rates of rhizome elongation and segment formation were observed in spring and summer when, in situ, plants were exposed to optimal environmental conditions for photosynthesis. This finding may be related to the seasonal capacity of temperate seagrasses to accumulate and store excess of energy produced from photosynthesis as carbohydrates in rhizomes in more suitable environmental conditions (e.g., Hemminga, 1998; Alcoverro et al., 2001). Such high-energy compounds can later support flower and seed production or act as an internal carbon source in less favorable environmental conditions in winter (e.g., Govers et al., 2015). On the other hand, the significantly higher leaf production observed in plants incubated in January-February might represent a mechanism to adjust photosynthetic structures to more suitable environmental conditions in spring. In contrast to this, plants incubated in November displayed the lowest leaf and rhizome growth rates which may represent a physiological acclimation to less suitable growth conditions in winter (e.g., Orth and Moore, 1986; Olesen and Sand-Jensen, 1993). There was evidence that seasonality modulated thermal and irradiance responses. For example, the growth performance of plants collected in April (in situ temperature at $12^{\circ} \mathrm{C}$ ) was less negatively impacted by extreme warming $\left(24^{\circ} \mathrm{C}\right)$ than plants collected in summer (in situ temperature $16^{\circ} \mathrm{C}$ ), particularly under low light conditions. Two further aspects suggest that seasonal acclimation modulates the observed experimental thermal responses: (i) highest mortality was observed in January (winter) while in July (summer) all plants survived; (ii) there were contrasting growth responses observed in plants incubated in April and November: in situ environmental conditions at these times of the year are very similar, however, growth rates in autumn were nearly half of those observed during experiments conducted in spring.

The flowering of $Z$. marina shoots observed in January at $12^{\circ} \mathrm{C}$ suggests that warming in cold seasons may advance flowering events in temperate seagrasses. Recent field studies reported that flowering in Irish $Z$. marina populations in situ currently starts in early March-April $\left(11-12^{\circ} \mathrm{C}\right)$ and ends in October-November (Beca-Carretero et al., 2019b). Previous studies also reported that anomalous warming can alter reproductive processes by increasing seed production in seagrasses (Ruiz et al., 2018; Qin et al., 2020a). Overall, year-round effects of climate change can 
lead to an internal decoupling of physiological and biochemical adjustments in marine primary producers (Bokhorst et al., 2009). For example, the accumulation of energy reserves such as non-structural carbohydrates may be affected, limiting their accumulation and impairing plant survival in cold winter periods (Alcoverro et al., 2001). Similarly, changes in temperature can affect flower production, flower fertilization and consequently seed production and germination (Qin et al., 2020a,b).

Detailed experimental studies that have investigated environmental stress on plants across different seasons are scarce and are mostly limited to terrestrial systems. For example, De Boeck et al. (2011) reported different sensitivities and responses to extreme events in terrestrial plants, dependent on the seasonal timing of the experiment. Our findings clearly demonstrate that, for temperate seagrasses, the time of year when temperature exposure occurs contributes to the observed growth, biochemical and survival responses. These results have relevant implications for future experimental design and interpretation of climate change effects on seagrasses. Nevertheless, caution is needed when extrapolating laboratory-derived results to natural habitats due to the complexity and the synergic interaction of several environmental parameters co-occurring in natural environments (De Boeck et al., 2010). Clearly, additional investigations are necessary to capture seasonal responses more comprehensively.

\section{Effects of Experimental Warming on the Nutritional Value of Seagrasses Leaves}

Particular nutritionally valuable compounds in seagrasses comprise essential fatty acids, carbohydrates, proteins or pigments, alongside secondary metabolites like phenolic compounds (e.g., Wang and Zheng, 2001; Pradheeba et al., 2011; Ahmed and Stepp, 2016).

The detailed review of the literature, together with experimental evidence from the present study, reveal sometimes contradicting trends pointing toward differential responses of specific biochemical compounds to increasing temperature. For instance, in some studies (e.g., Marín-Guirao et al., 2018; Artika et al., 2020), carbohydrates and photosynthetic pigments significantly increased in seagrass leaves at elevated temperatures; however, other studies report a significant decrease (e.g., Egea et al., 2018). Similar contrasting observations are documented for proteins, carbon, nitrogen and phosphorous contents (e.g., Hernán et al., 2017; Nguyen et al., 2020a; Viana et al., 2020; Table 2 and Supplementary Tables 14, 15). These differential patterns are likely to be linked to individual species-specific thermal tolerances, different degrees and duration of warming experiments or experimental time of year; they highlight an important gap in research into the effect of warming on the nutritional value of seagrasses. Common to all studies was the increase in necrotic leaf area under increased temperatures.

Our experimental results clearly demonstrate that warming significantly reduces omega-3 (n-3) PUFA levels, and favors the accumulation of SFA (see also Beca-Carretero et al., 2018b, 2020), as previously reported for other marine primary producers such as micro- and macroalgae (e.g., Gladyshev et al., 2013;
Aussant et al., 2018). PUFAs play a key role in regulating the membrane fluidity, electron transport and photosynthetic activity in the thylakoids of the chloroplast; hence, the reported lower levels of FA saturation in photosynthetic structures with increases in temperature could be expected (e.g., Sanina et al., 2008; Gosch et al., 2015; Sijil et al., 2019). Changes in FA levels and composition have previously been implemented as a sensitive indicator of responses of seagrasses, saltmarshes and algae to different environmental settings including temperature, irradiance or salinity (Duarte et al., 2018; Kostetsky et al., 2018; Beca-Carretero et al., 2020).

Climate change is expected to increase metabolic demands of seagrass herbivores (Burnell et al., 2013) and thus affect their nutritional preferences (de los Santos et al., 2012; Hernán et al., 2016; Pagès et al., 2018). Recent studies have demonstrated that changes in the biochemical and nutritional composition of terrestrial and marine primary producers induced by climate change effects are impacting trophic interactions between plants and consumers and their feeding behavior (O'Connor, 2009; Prado et al., 2010; Hoover et al., 2012; Hernán et al., 2016). Herbivore feeding experiments should be undertaken to evaluate the potential effects of diets with reduced $n-3$ PUFA contents, simulating future warming conditions.

\section{Ecological Significance}

Under global warming scenarios marine heatwaves are predicted to increase in duration, intensity and frequency but also can occur across different seasons including in colder periods (Belkin, 2009; Frölicher et al., 2018). Our experimental results are thus ecologically highly relevant as they indicate that warming will not only affect seagrass productivity and physiology but also their reproductive effort. Conventionally, climate experiments and particularly warming assessment (review in Beca-Carretero et al., 2018b), have been conducted in warmer months, however, our study highlighted the importance of conducting climate change experiments across all seasons. In addition, our outcomes showed the higher vulnerability of plants exposed to low levels of irradiance under warming episodes. This is particularly important in the context of expected increases and variability of extreme events such as storms and extreme warming events associated with global change, resulting in synergetic stresses in shallow marine habitats (Lefcheck et al., 2017). Nevertheless, our experiments only represent short-time simulations such as marine heatwaves, and complementary experiments simulating long-term effects of warming and light stress are necessary to complement such an approach. Additionally, the magnitude of the selected range of temperatures, irradiance treatments and the duration of the exposure would significantly affect seagrass responses to experimental warming (Nguyen et al., 2021). Overall, our results have important ecological implications for the adequate management of seagrass ecosystems to ensure a balance of optimal temperature and irradiance conditions; they also point toward the critical importance to develop all-year monitoring programs in order to capture more comprehensively the potential effects of climate change events across all seasons. 
Lastly, our results demonstrated that warming will dismiss the capacity of temperate seagrasses to produce and accumulate the essential omega-3 PUFA. These observations can have critical biological consequences as omega- 3 are essential FAs that cannot be synthetized by herbivores and are transferred throughout the trophic chain (Sargent et al., 1999; Tocher, 2003; Parrish, 2009). Besides the direct ecological impact on herbivores, a reduction in omega-3 PUFA in seagrasses in response to climate change may also reduce the nutritional quality of some marine food sources (fishes or crustaceans) for human consumption (e.g., Kang, 2011; Hixson and Arts, 2016).

\section{CONCLUSION}

Our results demonstrate that responses of temperate seagrasses to experimental temperature and light stress are partially modulated by the seasonal acclimatization during an annual cycle: the time of year at which experiments are conducted strongly influences the observed growth, physiological and survival responses. These finding have highly relevant implications for experimental design, and in the understanding and interpretation of the effects of experimental warming on seagrasses. Increases in sea surface temperature (SST) predicted for the end of this century are expected to favor the growth and production of temperate seagrasses growing under temperature-limited conditions; by contrast, light-limited plants will be more vulnerable to thermal stress. Additionally, warming will affect the biochemical composition and associated nutritional quality of seagrass leaves with a potential effect on herbivores and its associated trophic change.

\section{DATA AVAILABILITY STATEMENT}

The original contributions presented in the study are included in the article/Supplementary Material, further inquiries can be directed to the corresponding author/s.

\section{REFERENCES}

Ahmed, S., and Stepp, J. R. (2016). Beyond yields: climate effects on specialty crop quality and agroecological management. Elem. Sci. Anth. 4:92.

Alcoverro, T., Manzanera, M., and Romero, J. (2001). Annual metabolic carbon balance of the seagrass Posidonia oceanica: the importance of carbohydrate reserves. Mar. Ecol. Prog. Ser. 211, 105-116. doi: 10.3354/meps 211105

Aoki, L. R., McGlathery, K. J., Wiberg, P. L., and Al-Haj, A. (2020). Depth affects seagrass restoration success and resilience to marine heat wave disturbance. Estuaries Coast. 43, 316-328. doi: 10.1007/s12237-019-00685-0

Artika, S. R., Ambo-Rappe, R., Teichberg, M., Moreira-Saporiti, A., and Viana, I. G. (2020). Morphological and physiological responses of Enhalus acoroides seedlings under varying temperature and nutrient treatment. Front. Mar. Sci. 7:325. doi: $10.3389 /$ fmars.2020.00325

Aussant, J., Guihéneuf, F., and Stengel, D. B. (2018). Impact of temperature on fatty acid composition and nutritional value in eight species of microalgae. Appl. Microbiol. Biotechnol. 102, 5279-5297. doi: 10.1007/s00253-018-9001-x

Beca-Carretero, P., Guihéneuf, F., Krause-Jensen, D., and Stengel, D. B. (2020). Seagrass fatty acid profiles as a sensitive indicator of climate settings across seasons and latitudes. Mar. Env. Res. 161:105075. doi: 10.1016/j.marenvres. 2020.105075

\section{AUTHOR CONTRIBUTIONS}

PB-C and DS developed the experimental design. PB-C, MJ-M, $\mathrm{CS}$, and FG collected the samples and conducted the laboratory experiments at NUIG. TA-G, FG, CS, and PB-C performed the laboratory analysis. $\mathrm{PB}-\mathrm{C}$ performed the statistical analysis, prepared a first draft of the manuscript. DS provided resources and funding for the project. All co-authors commented on and provided edits to finalize the original manuscript. All coauthors are in agreement with the submission of the final manuscript. All authors contributed to the article and approved the submitted version.

\section{FUNDING}

This project was supported by a College of Science (NUI Galway) Scholarship to PB-C. This research was additionally supported by the VOCAB project (Grant-Aid Agreement No. PBA/ME/16/01) funded by the Marine Institute under the Marine Research Programme by the Irish Government.

\section{ACKNOWLEDGMENTS}

We are very grateful to the Associate Editor and the reviewers for their valuable comments and constructive criticisms during the review process.

\section{SUPPLEMENTARY MATERIAL}

The Supplementary Material for this article can be found online at: https://www.frontiersin.org/articles/10.3389/fmars. 2021.731152/full\#supplementary-material

Beca-Carretero, P., Guihéneuf, F., Marín-Guirao, L., Bernardeau-Esteller, J., García-Muñoz, R., Stengel, D. B., et al. (2018a). Effects of an experimental heat wave on fatty acid composition in two Mediterranean seagrass species. Mar. Pollut. Bull. 134, 27-37. doi: 10.1016/j.marpolbul.2017.12.057

Beca-Carretero, P., Guihéneuf, F., Winters, G., and Stengel, D. B. (2019a). Depthinduced adjustment of fatty acid and pigment composition suggests high biochemical plasticity in the tropical seagrass Halophila stipulacea. Mar. Ecol. Prog. Ser. 608, 105-117. doi: 10.3354/meps 12816

Beca-Carretero, P., Olesen, B., Marbà, N., and Krause-Jensen, D. (2018b). Response to experimental warming in northern eelgrass populations: comparison across a range of temperature adaptations. Mar. Ecol. Prog. Ser. 589, 59-72. doi: 10.3354/meps12439

Beca-Carretero, P., Stanschewski, C. S., Julia-Miralles, M., Sanchez-Gallego, A., and Stengel, D. B. (2019b). Temporal and depth-associated changes in the structure, morphometry and production of near-pristine Zostera marina meadows in western Ireland. Aquat. Bot. 155, 5-17. doi: 10.1016/j.aquabot.2019.02.003

Belkin, I. M. (2009). Rapid warming of large marine ecosystems. Prog. Oceanogr. 81, 207-213. doi: 10.1016/j.pocean.2009.04.011

Bennett, S., Wernberg, T., Joy, B. A., De Bettignies, T., and Campbell, A. H. (2015). Central and rear-edge populations can be equally vulnerable to warming. Nat. Commun. 6:10280. 
Bertelli, C. M., and Unsworth, R. K. (2018). Light stress responses by the eelgrass, Zostera marina (L). Front. Environ. Sci. 6:39. doi: 10.3389/fenvs.2018.00039

Bjerke, J. W., Bokhorst, S., Zielke, M., Callaghan, T. V., Bowles, F. W., and Phoenix, G. K. (2011). Contrasting sensitivity to extreme winter warming events of dominant sub-Arctic heathland bryophyte and lichen species. J. Ecol. 99, 1481-1488. doi: 10.1111/j.1365-2745.2011.01859.x

Bokhorst, S. F., Bjerke, J. W., Tømmervik, H., Callaghan, T. V., and Phoenix, G. K. (2009). Winter warming events damage sub-Arctic vegetation: consistent evidence from an experimental manipulation and a natural event. J. Ecol. 97, 1408-1415. doi: 10.1111/j.1365-2745.2009.01554.x

Boutahar, L., Espinosa, F., Richir, J., Lepoint, G., Gobert, S., Maanan, M., et al. (2020). Deep-water Zostera marina meadows in the Mediterranean. Aquat. Bot. 166:103269. doi: 10.1016/j.aquabot.2020.103269

Burnell, O. W., Russell, B. D., Irving, A. D., and Connell, S. D. (2013). Eutrophication offsets increased sea urchin grazing on seagrass caused by ocean warming and acidification. Mar. Ecol. Prog. Ser. 485, 37-46. doi: 10.3354/ meps10323

Carruthers, T. J. B., Dennison, W. C., Kendrick, G. A., Waycott, M., Walker, D. I., and Cambridge, M. L. (2007). Seagrasses of south-west Australia: a conceptual synthesis of the world's most diverse and extensive seagrass meadows. J. Exp. Mar. Biol. Ecol. 350, 21-45. doi: 10.1016/j.jembe.2007.05.036

Chartrand, K. M., Szabó, M., Sinutok, S., Rasheed, M. A., and Ralph, P. J. (2018). Living at the margins-the response of deep-water seagrasses to light and temperature renders them susceptible to acute impacts. Mar. Environ. Res. 136, 126-138. doi: 10.1016/j.marenvres.2018.02.006

Collier, C. J., and Waycott, M. (2014). Temperature extremes reduce seagrass growth and induce mortality. Mar. Pollut. Bull. 83, 483-490. doi: 10.1016/j. marpolbul.2014.03.050

Cott, G., Beca-Carretero, P., and Stengel, D. B. (2021). Blue Carbon and Marine Carbon Sequestration in Irish Waters and Coastal Habitats. Galway: Marine Institute.

Dale, A. L., McAllen, R., and Whelan, P. (2007). Management Considerations for Subtidal Zostera marina Beds in Ireland. Dublin, Ireland: Department of Environment, Heritage and Local Government. National Parks and Wildlife Service.

De Boeck, H. J., Dreesen, F. E., Janssens, I. A., and Nijs, I. (2010). Climatic characteristics of heat waves and their simulation in plant experiments. Glob. Chang. Biol. 16, 1992-2000. doi: 10.1111/j.1365-2486.2009.02049.x

De Boeck, H. J., Dreesen, F. E., Janssens, I. A., and Nijs, I. (2011). Whole-system responses of experimental plant communities to climate extremes imposed in different seasons. New Phytol. 189, 806-817. doi: 10.1111/j.1469-8137.2010. 03515.x

de los Santos, C. B., Brun, F. G., Onoda, Y., Cambridge, M. L., Bouma, T. J., Vergara, J. J., et al. (2012). Leaf-fracture properties correlated with nutritional traits in nine Australian seagrass species: implications for susceptibility to herbivory. Mar. Ecol. Prog. Ser. 458, 89-102. doi: 10.3354/meps09757

de los Santos, C. B., Krause-Jensen, D., Alcoverro, T., Marbà, N., Duarte, C. M., Van Katwijk, M. M., et al. (2019). Recent trend reversal for declining European seagrass meadows. Nat. Commun. 10:3356.

Duarte, B., Martins, I., Rosa, R., Matos, A. R., Roleda, M. Y., Reusch, T. B., et al. (2018). Climate change impacts on seagrass meadows and macroalgal forests: an integrative perspective on acclimation and adaptation potential. Front. Mar Sci. 5:190. doi: 10.3389/fmars.2018.00190

Duarte, C. M. (2002). The future of seagrass meadows. Environ. Conserv. 29, 192-206. doi: 10.1017/s0376892902000127

Duarte, C. M., and Kalff, J. (1987). Latitudinal influences on depths of maximum colonization and maximum biomass of submerged angiosperms in lakes. Can. J. Fish. Aquat. Sci. 44, 1759-1764. doi: 10.1139/f87-215

Dubois, K., Williams, S. L., and Stachowicz, J. J. (2020). Previous exposure mediates the response of eelgrass to future warming via clonal transgenerational plasticity. Ecology 101:e03169.

Egea, L. G., Jiménez-Ramos, R., Vergara, J. J., Hernández, I., and Brun, F. G. (2018). Interactive effect of temperature, acidification and ammonium enrichment on the seagrass Cymodocea nodosa. Mar. Pollut. Bull. 134, 14-26. doi: 10.1016/j. marpolbul.2018.02.029

Evans, A. S., Webb, K. L., and Penhale, P. A. (1986). Photosynthetic temperature acclimation in two coexisting seagrasses, Zostera marina L. and Ruppia maritima L. Aquat. Bot. 24, 185-197. doi: 10.1016/0304-3770(86)90095-1
Falcone, D. L., Ogas, J. P., and Somerville, C. R. (2004). Regulation of membrane fatty acid composition by temperature in mutants of Arabidopsis with alterations in membrane lipid composition. BMC Plant Biol. 4:17. doi: 10.1186/ 1471-2229-4-17

Feudale, L., and Shukla, J. (2011). Influence of sea surface temperature on the European heat wave of 2003 summer. Part I: an observational study. Clim. Dyn. 36, 1691-1703. doi: 10.1007/s00382-010-0788-0

Frölicher, T. L., Fischer, E. M., and Gruber, N. (2018). Marine heatwaves under global warming. Nature 560, 360-364. doi: 10.1038/s41586-018-0383-9

Gao, Y., Fang, J., Du, M., Fang, J., Jiang, W., and Jiang, Z. (2017). Response of the eelgrass (Zostera marina L.) to the combined effects of high temperatures and the herbicide, atrazine. Aquat. Bot. 142, 41-47. doi: 10.1016/j.aquabot.2017. 06.005

Gladyshev, M. I., Sushchik, N. N., and Makhutova, O. N. (2013). Production of EPA and DHA in aquatic ecosystems and their transfer to the land. Prostaglandins Other Lipid Mediat. 107, 117-126. doi: 10.1016/j.prostaglandins.2013.03.002

Gombos, Z., Wada, H., Hideg, E., and Murata, N. (1994). The unsaturation of membrane lipids stabilizes photosynthesis against heat stress. Plant Physiol. 104, 563-567. doi: 10.1104/pp.104.2.563

Gosch, B. J., Paul, N. A., de Nys, R., and Magnusson, M. (2015). Seasonal and within-plant variation in fatty acid content and composition in the brown seaweed Spatoglossum macrodontum (Dictyotales, Phaeophyceae). J. Appl. Phycol. 27, 387-398. doi: 10.1007/s10811-014-0308-4

Govers, L. L., Suykerbuyk, W., Hoppenreijs, J. H., Giesen, K., Bouma, T. J., and van Katwijk, M. M. (2015). Rhizome starch as indicator for temperate seagrass winter survival. Ecol. Indic. 49, 53-60. doi: 10.1016/j.ecolind.2014.10.002

Guihéneuf, F., and Stengel, D. B. (2013). LC-PUFA-enriched oil production by microalgae: accumulation of lipid and triacylglycerols containing n-3 LC-PUFA is triggered by nitrogen limitation and inorganic carbon availability in the marine haptophyte Pavlova lutheri. Mar. Drugs 11, 4246-4266. doi: 10.3390/ md11114246

Hastings, R., Cummins, V., and Holloway, P. (2020). Assessing the impact of physical and anthropogenic environmental factors in determining the habitat suitability of seagrass ecosystems. Sustainability 12:8302. doi: 10.3390/ su12208302

Hemminga, M. A. (1998). The root/rhizome system of seagrasses: an asset and a burden. J. Sea Res. 39, 183-196. doi: 10.1016/s1385-1101(98)00004-5

Hemminga, M. A., and Duarte, C. M. (2000). Seagrass Ecology. Cambridge: Cambridge University Press.

Hernán, G., Castejón, I., Terrados, J., and Tomas, F. (2019). Herbivory and resource availability shift plant defense and herbivore feeding choice in a seagrass system. Oecologia 189, 719-732. doi: 10.1007/s00442-019-04364-6

Hernán, G., Ortega, M. J., Gándara, A. M., Castejón, I., Terrados, J., and Tomas, F. (2017). Future warmer seas: increased stress and susceptibility to grazing in seedlings of a marine habitat-forming species. Glob. Chang. Biol. 23, 4530-4543. doi: $10.1111 / \mathrm{gcb} .13768$

Hernán, G., Ramajo, L., Basso, L., Delgado, A., Terrados, J., Duarte, C. M., et al. (2016). Seagrass (Posidonia oceanica) seedlings in a high-CO2 world: from physiology to herbivory. Sci. Rep. 6:38017.

Hixson, S. M., and Arts, M. T. (2016). Climate warming is predicted to reduce omega-3, long-chain, polyunsaturated fatty acid production in phytoplankton. Glob. Change Biol. 22, 2744-2755. doi: 10.1111/gcb.13295

Hobday, A. J., Alexander, L. V., Perkins, S. E., Smale, D. A., Straub, S. C., Oliver, E. C. J., et al. (2016). A hierarchical approach to defining marine heatwaves. Prog. Oceanogr. 141, 227-238. doi: 10.1016/j.pocean.2015. 12.014

Höffle, H., Thomsen, M. S., and Holmer, M. (2011). High mortality of Zostera marina under high temperature regimes but minor effects of the invasive macroalgae Gracilaria vermiculophylla. Estuar. Coast. Shelf Sci. 92, 35-46. doi: 10.1016/j.ecss.2010.12.017

Hoover, S. E., Ladley, J. J., Shchepetkina, A. A., Tisch, M., Gieseg, S. P., and Tylianakis, J. M. (2012). Warming, CO2, and nitrogen deposition interactively affect a plant-pollinator mutualism. Ecol. Lett. 15, 227-234. doi: 10.1111/j.14610248.2011.01729.x

IPCC (2014). "Climate change 2014: Synthesis Report," in Core Writing Team, Contribution of Working Group II to the 5th Assessment Report of the Intergovernmental Panel on Climate Change, eds R. K. Pachauri and L. A. Meyer (Geneva: IPCC), 151. 
Johnson, A. J., Shields, E. C., Kendrick, G. A., and Orth, R. J. (2021). Recovery dynamics of the seagrass Zostera marina following mass mortalities from two extreme climatic events. Estuaries. Coast. 44, 535-544. doi: 10.1007/s12237020-00816-y

Jorda, G., Marbà, N., Bennett, S., Santana-Garcon, J., Agusti, S., and Duarte, C. M. (2020). Ocean warming compresses the three-dimensional habitat of marine life. Nat. Ecol. Evol. 4, 109-114. doi: 10.1038/s41559-019-1058-0

Kang, J. X. (2011). Omega-3: a link between global climate change and human health. Biotechnol. Adv. 29, 388-390. doi: 10.1016/j.biotechadv.2011.02.003

Kim, D. H., Mahomoodally, M. F., Sadeer, N. B., Seok, P. G., Zengin, G., Palaniveloo, K., et al. (2021). Nutritional and bioactive potential of seagrasses: a review. S. Afr. J. Bot. 137, 216-227. doi: 10.1016/j.sajb.2020.10.018

Koch, M., Bowes, G., Ross, C., and Zhang, X. H. (2013). Climate change and ocean acidification effects on seagrasses and marine macroalgae. Glob. Change Biol. $19,103-132$.

Koch, M. S., Schopmeyer, S., Kyhn-Hansen, C., and Madden, C. J. (2007). Synergistic effects of high temperature and sulfide on tropical seagrass. J. Exp. Mar. Biol. Ecol. 341, 91-101. doi: 10.1016/j.jembe.2006.10.004

Kostetsky, E., Chopenko, N., Barkina, M., Velansky, P., and Sanina, N. (2018). Fatty acid composition and thermotropic behavior of glycolipids and other membrane lipids of Ulva lactuca (Chlorophyta) inhabiting different climatic zones. Mar. Drugs 16:494. doi: 10.3390/md16120494

Krause-Jensen, D., Carstensen, J., Nielsen, S. L., Dalsgaard, T., Christensen, P. B., Fossing, H., et al. (2011). Sea bottom characteristics affect depth limits of eelgrass Zostera marina. Mar. Ecol. Prog. Ser. 425, 91-102. doi: 10.3354/ meps09026

Krause-Jensen, D., and Duarte, C. M. (2014). Expansion of vegetated coastal ecosystems in the future Arctic. Front. Mar. Sci. 1:77. doi: 10.3389/fmars.2014. 00077

Krause-Jensen, D., Duarte, C. M., Hendriks, E. G., Meire, L., Blicher, M. E., Marbà, N., et al. (2015). Macroalgae contribute to nested mosaics of $\mathrm{pH}$ variability in a subarctic fjord. Biogeosciences 12, 4895-4911. doi: 10.5194/bg-12-4895-2015

Krause-Jensen, D., Greve, T. M., and Nielsen, K. (2005). Eelgrass as a bioindicator under the European water framework directive. Water Resour. Manag. 19, 63-75. doi: 10.1007/s11269-005-0293-0

Krause-Jensen, D., Marbà, N., Olesen, B., Sejr, M. K., Christensen, P. B., Rodrigues, J., et al. (2012). Seasonal sea ice cover as principal driver of spatial and temporal variation in depth extension and annual production of kelp in Greenland. Glob. Chang. Biol. 18, 2981-2994. doi: 10.1111/j.1365-2486.2012.02765.x

Lee, K. S., and Dunton, K. H. (1997). Effect of in situ light reduction on the maintenance, growth and partitioning of carbon resources in Thalassia testudinum banks ex König. J. Exp. Mar. Biol. Ecol. 210, 53-73. doi: 10.1016/ s0022-0981(96)02720-7

Lee, K. S., Park, S. R., and Kim, J. B. (2005). Production dynamics of the eelgrass, Zostera marina in two bay systems on the south coast of the Korean peninsula. Mar. Biol. 147, 1091-1108. doi: 10.1007/s00227-005-0011-8

Lee, K. S., Park, S. R., and Kim, Y. K. (2007). Effects of irradiance, temperature, and nutrients on growth dynamics of seagrasses: a review. J. Exp. Mar. Biol. Ecol. 350, 144-175. doi: 10.1016/j.jembe.2007.06.016

Lefcheck, J. S., Wilcox, D. J., Murphy, R. R., Marion, S. R., and Orth, R. J. (2017). Multiple stressors threaten the imperiled coastal foundation species eelgrass (Zostera marina) in Chesapeake Bay, USA. Glob. Chang. Biol. 23, 3474-3483. doi: $10.1111 / \mathrm{gcb} .13623$

Lichtenthaler, H. K., and Wellburn, A. R. (1983). Determination of total carotenoids and chlorophyll a and b of leaf extract in different solvents. Biochem. Soc. Trans. 603, 591-592. doi: 10.1042/bst0110591

Longstaff, B. J., and Dennison, W. C. (1999). Seagrass survival during pulsed turbidity events: the effects of light deprivation on the seagrasses Halodule pinifolia and Halophila ovalis. Aquat. Bot. 65, 105-121. doi: 10.1016/s03043770(99)00035-2

Lopez-Calderon, J. M., Riosmena-Rodríguez, R., Torre, J., Meling, A., and Basurto, X. (2016). Zostera marina meadows from the Gulf of California: conservation status. Biodivers. Conserv. 25, 261-273. doi: 10.1007/s10531-016$1045-6$

Macreadie, P. I., Anton, A., Raven, J. A., Beaumont, N., Connolly, R. M., Friess, D. A., et al. (2019). The future of blue carbon science. Nat. Commun. 10, 1-13.

Madden, B., Jennings, E., and Jeffrey, D. W. (1993). Distribution and ecology of Zostera in Co. Dublin. Ir. Nat. J. 24, 303-310.
Marbà, N., and Duarte, C. M. (2010). Mediterranean warming triggers seagrass (Posidonia oceanica) shoot mortality. Glob. Chang. Biol. 16, 2366-2375. doi: $10.1111 / j .1365-2486.2009 .02130 . x$

Marín-Guirao, L., Bernardeau-Esteller, J., García-Muñoz, R., Ramos, A., Ontoria, Y., Romero, J., et al. (2018). Carbon economy of Mediterranean seagrasses in response to thermal stress. Mar. Pollut. Bull. 135, 617-629. doi: 10.1016/j. marpolbul.2018.07.050

Morita, T., Okumura, H., Abe, M., Kurashima, A., and Maegawa, M. (2007). Density and distribution of seeds in bottom sediments in Zostera marina beds in Ago Bay, central Japan. Aquat. Bot. 87, 38-42. doi: 10.1016/j.aquabot.2007. 03.001

Mvungi, E. F., and Pillay, D. (2019). Eutrophication overrides warming as a stressor for a temperate African seagrass (Zostera capensis). PLoS One 14:e0215129. doi: 10.1371/journal.pone.0215129

Nguyen, H. M., Kim, M., Ralph, P. J., Marín-Guirao, L., Pernice, M., and Procaccini, G. (2020b). Stress memory in seagrasses: first insight into the effects of thermal priming and the role of epigenetic modifications. Front. Plant Sci. 11:494. doi: $10.3389 /$ fpls.2020.00494

Nguyen, H. M., Ralph, P. J., Marín-Guirao, L., Pernice, M., and Procaccini, G. (2021). Seagrasses in an era of ocean warming: a review. Biol. Rev. Camb. Philos. Soc. 96, 2009-2030. doi: 10.1111/brv.12736

Nguyen, H. M., Yadav, N. S., Barak, S., Lima, F. P., Sapir, Y., and Winters, G. (2020a). Responses of invasive and native populations of the seagrass Halophila stipulacea to simulated climate change. Front. Mar. Sci. 6:812. doi: 10.3389/ fmars.2019.00812

Niu, S., Zhang, P., Liu, J., Guo, D., and Zhang, X. (2012). The effect of temperature on the survival, growth, photosynthesis, and respiration of young seedlings of eelgrass Zostera marina L. Aquaculture 350, 98-108. doi: 10.1016/j.aquaculture. 2012.04.010

Nordlund, L. M., Jackson, E. L., Nakaoka, M., Samper-Villarreal, J., BecaCarretero, P., and Creed, J. C. (2018). Seagrass ecosystem services-what's next? Mar. Pollut. Bull. 134, 145-151. doi: 10.1016/j.marpolbul.2017. 09.014

NPWS (2014). Kilkieran Bay and Islands SAC (site Code: 2111) Conservation objectives supporting document-marine habitats and species (unpublished). Available online at: https://www.npws.ie.

O'Connor, M. I. (2009). Warming strengthens an herbivore-plant interaction. Ecology 90, 388-398. doi: 10.1890/08-0034.1

Olesen, B., Enríquez, S., Duarte, C. M., and Sand-Jensen, K. (2002). Depthacclimation of photosynthesis, morphology and demography of Posidonia oceanica and Cymodocea nodosa in the Spanish Mediterranean Sea. Mar. Ecol. Prog. Ser. 236, 89-97. doi: 10.3354/meps236089

Olesen, B., Krause-Jensen, D., Marbà, N., and Christensen, P. B. (2015). Eelgrass Zostera marina in subarctic Greenland: dense meadows with slow biomass turnover in cold waters. Mar. Ecol. Prog. Ser. 518, 107-121. doi: 10.3354/ meps 11087

Olesen, B., and Sand-Jensen, K. (1993). Seasonal acclimatization of eelgrass Zostera marina growth to light. Mar. Ecol. Prog. Ser. 94, 91-99. doi: 10.3354/ meps094091

Ondiviela, B., Galván, C., Recio, M., Jiménez, M., Juanes, J. A., Puente, A., et al. (2020). Vulnerability of Zostera noltei to sea level rise: the use of clustering techniques in climate change studies. Estuaries. Coast. 43, 2063-2075. doi: 10.1007/s12237-020-00742-z

Ondiviela, B., Losada, I. J., Lara, J. L., Maza, M., Galván, C., Bouma, T. J., et al. (2014). The role of seagrasses in coastal protection in a changing climate. Coast. Eng. 87, 158-168. doi: 10.1016/j.coastaleng.2013.11.005

Ontoria, Y., Cuesta-Gracia, A., Ruiz, J. M., Romero, J., and Pérez, M. (2019a). The negative effects of short-term extreme thermal events on the seagrass Posidonia oceanica are exacerbated by ammonium additions. PLoS One 14:e0222798. doi: 10.1371/journal.pone.0222798

Ontoria, Y., Gonzalez-Guedes, E., Sanmartí, N., Bernardeau-Esteller, J., Ruiz, J. M., Romero, J., et al. (2019b). Interactive effects of global warming and eutrophication on a fast-growing Mediterranean seagrass. Mar. Environ. Res. 145, 27-38. doi: 10.1016/j.marenvres.2019.02.002

Ontoria, Y., Webster, C., Said, N., Ruiz, J. M., Pérez, M., Romero, J., et al. (2020). Positive effects of high salinity can buffer the negative effects of experimental warming on functional traits of the seagrass Halophila ovalis. Mar. Pollut. Bull. 158:111404. doi: 10.1016/j.marpolbul.2020.111404 
Orth, R. J., Carruthers, T. J. B., Dennison, W. C., Duarte, C. M., Fourqurean, J. W., Heck, K. L., et al. (2006). A global crisis for seagrass ecosystems. Bioscience 56, 987-996.

Orth, R. J., and Moore, K. A. (1986). Seasonal and year-to-year variations in the growth of Zostera marina L.(eelgrass) in the lower Chesapeake Bay. Aquat. Bot. 24, 335-341. doi: 10.1016/0304-3770(86)90100-2

Pagès, J. F., Smith, T. M., Tomas, F., Sanmarti, N., Boada, J., De Bari, H., et al. (2018). Contrasting effects of ocean warming on different components of plantherbivore interactions. Mar. Pollut. Bull. 134, 55-65. doi: 10.1016/j.marpolbul. 2017.10.036

Parrish, C. C. (2009). "Essential fatty acids in aquatic food webs," in Lipids in Aquatic Ecosystems, eds M. Kainz, M. Brett, and M. Arts (New York, NY: Springer), 309-326. doi: 10.1007/978-0-387-89366-2_13

Pazzaglia, J., Santillán-Sarmiento, A., Helber, S. B., Ruocco, M., Terlizzi, A., MarínGuirao, L., et al. (2020). Does warming enhance the effects of eutrophication in the seagrass Posidonia oceanica? Front. Mar. Sci. 7:1067. doi: 10.3389/fmars. 2020.564805

Pereda-Briones, L., Terrados, J., and Tomas, F. (2019). Negative effects of warming on seagrass seedlings are not exacerbated by invasive algae. Mar. Pollut. Bull. 141, 36-45. doi: 10.1016/j.marpolbul.2019.01.049

Phillips, R. C. (1974). Transplantation of seagrasses, with special emphasis on eelgrass, Zostera marina L. Aquaculture 4, 161-176. doi: 10.1016/00448486(74)90031-3

Pradheeba, M., Dilipan, E., Nobi, E. P., Thangaradjou, T., and Sivakumar, K. (2011). Evaluation of seagrasses for their nutritional value. Indian J. Geo-Mar. Sci. 40, 105-111.

Prado, P., Romero, J., and Alcoverro, T. (2010). Nutrient status, plant availability and seasonal forcing mediate fish herbivory in temperate seagrass beds. Mar. Ecol. Prog. Ser. 409, 229-239. doi: 10.3354/meps08585

Qin, L. Z., Kim, S. H., Song, H. J., Kim, H. G., Suonan, Z., Kwon, O., et al. (2020a). Long-term variability in the flowering phenology and intensity of the temperate seagrass Zostera marina in response to regional sea warming. Ecol. Indic. 119:106821. doi: 10.1016/j.ecolind.2020.106821

Qin, L. Z., Kim, S. H., Song, H. J., Suonan, Z., Kim, H., Kwon, O., et al. (2020b). Influence of regional water temperature variability on the flowering phenology and sexual reproduction of the seagrass Zostera marina in Korean coastal waters. Estuaries Coast. 43, 449-462. doi: 10.1007/s12237-019-00569-3

Ralph, P. J., Durako, M. J., Enríquez, S., Collier, C. J., and Doblin, M. A. (2007). Impact of light limitation on seagrasses. J. Exp. Mar. Biol. Ecol. 350, 176-193. doi: 10.1016/j.jembe.2007.06.017

Repolho, T., Duarte, B., Dionísio, G., Paula, J. R., Lopes, A. R., Rosa, I. C., et al. (2017). Seagrass ecophysiological performance under ocean warming and acidification. Sci. Rep. 7:41443.

Reusch, T. B. H., Ehlers, A., Hämmerli, A., and Worm, B. (2005). Ecosystem recovery after climatic extremes enhanced by genotypic diversity. Proc. Natl. Acad. Sci. U.S.A. 102, 2826-2831. doi: 10.1073/pnas.0500008102

Roca, G., Alcoverro, T., Krause-Jensen, D., Balsby, T. J., Van Katwijk, M. M., Marbà, N., et al. (2016). Response of seagrass indicators to shifts in environmental stressors: a global review and management synthesis. Ecol. Indic. 63, 310-323. doi: $10.1016 /$ j.ecolind.2015.12.007

Rooney, N., and Kalff, J. (2000). Inter-annual variation in submerged macrophyte community biomass and distribution: the influence of temperature and lake morphometry. Aquat. Bot. 68, 321-335. doi: 10.1016/s0304-3770(00)00 126-1

Ruiz, J. M., Marín-Guirao, L., García-Muñoz, R., Ramos-Segura, A., BernardeauEsteller, J., Pérez, M., et al. (2018). Experimental evidence of warming-induced flowering in the Mediterranean seagrass Posidonia oceanica. Mar. Pollut. Bull. 134, 49-54. doi: 10.1016/j.marpolbul.2017.10.037

Ruiz, J. M., and Romero, J. (2003). Effects of disturbances caused by coastal constructions on spatial structure, growth dynamics and photosynthesis of the seagrass Posidonia oceanica. Mar. Pollut. Bull. 46, 1523-1533. doi: 10.1016/j. marpolbul.2003.08.021

Sand-Jensen, K. (1975). Biomass, net production and growth dynamics in an eelgrass (Zostera marina L.) population in Vellerup Vig, Denmark. Ophelia 14, 185-201. doi: 10.1080/00785236.1975.10422501

Sanina, N. M., Goncharova, S. N., and Kostetsky, E. Y. (2008). Seasonal changes of fatty acid composition and thermotropic behaviour of polar lipids from marine macrophytes. Phytochemistry 69, 1517-1527. doi: 10.1016/j.phytochem.2008. 01.014

Sargent, J., McEvoy, L., Estevez, A., Bell, G., Bell, M., Henderson, J., et al. (1999). Lipid nutrition of marine fish during early development: current status and future directions. Aquaculture 179, 217-229. doi: 10.1016/s0044-8486(99) 00191-X

Schmid, M., Guihéneuf, F., and Stengel, D. B. (2014). Fatty acid contents and profiles of 16 macroalgae collected from the Irish coast at two seasons. J. Appl. Phycol. 26, 451-463. doi: 10.1007/s10811-013-0132-2

Short, F., Carruthers, T., Dennison, W., and Waycott, M. (2007). Global seagrass distribution and diversity: a bioregional model. J. Exp. Mar. Biol. Ecol. 350, 3-20. doi: 10.1016/j.jembe.2007.06.012

Short, F. T., and Duarte, C. M. (2001). "Methods for the measurement of seagrass growth and production," in Global Seagrass Research Methods, eds F. T. Short and R. G. Coles (Amsterdam: Elsevier), 155-182. doi: 10.1016/b978044450891-1/50009-8

Short, F. T., Koch, E. W., Creed, J. C., Magalhaes, K. M., Fernandez, E., and Gaeckle, J. L. (2006). SeagrassNet monitoring across the Americas: case studies of seagrass decline. Mar. Ecol. 27, 277-289. doi: 10.1111/j.1439-0485.2006.00095.x

Short, F. T., and Neckles, H. A. (1999). The effects of global climate change on seagrasses. Aquat. Bot. 63, 169-196. doi: 10.1016/s0304-3770(98)00117-x

Short, F. T., and Wyllie-Echeverria, S. (1996). Natural and human-induced disturbance of seagrasses. Environ. Conserv. 23, 17-27. doi: 10.1017/ s0376892900038212

Sijil, P. V., Sarada, R., and Chauhan, V. S. (2019). Enhanced accumulation of alphalinolenic acid rich lipids in indigenous freshwater microalga Desmodesmus sp.: the effect of low-temperature on nutrient replete, UV treated and nutrient stressed cultures. Bioresour. Technol. 273, 404-415. doi: 10.1016/j.biortech. 2018.11.028

Silva, J., Barrote, I., Costa, M. M., Albano, S., and Santos, R. (2013). Physiological responses of Zostera marina and Cymodocea nodosa to light-limitation stress. PLoS One 8:e81058. doi: 10.1371/journal.pone.0081058

Solovchenko, A. E., Khozin-Goldberg, I., Didi-Cohen, S., Cohen, Z., and Merzlyak, M. N. (2008). Effects of light intensity and nitrogen starvation on growth, total fatty acids and arachidonic acid in the green microalga Parietochloris incisa. J. Appl. Phycol. 20, 245-251. doi: 10.1007/s10811-007-9233-0

Staehr, P. A., and Borum, J. (2011). Seasonal acclimation in metabolism reduces light requirements of eelgrass (Zostera marina). J. Exp. Mar. Biol. Ecol. 407, 139-146. doi: 10.1016/j.jembe.2011.05.031

Stengel, D. B., Wilkes, R., and Guiry, M. (1999). Seasonal growth and recruitment of Himanthalia elongata Fucales, Phaeophycota) in different habitats on the Irish west coast. Eur. J. Phycol. 34, 213-221. doi: 10.1017/s0967026299 002115

Tinker, J. P., and Howes, E. L. (2020). The impacts of climate change on temperature (air and sea), relevant to the coastal and marine environment around the UK. MCCIP Sci. Rev. 2020, 1-3.

Tocher, D. R. (2003). Metabolism and functions of lipids and fatty acids in teleost fish. Rev. Fish. Sci. 11, 107-184. doi: 10.1080/713610925

Touchette, B. W., Burkholder, J. M., and Glasgow, H. B. (2003). Variations in eelgrass (Zostera marina L.) morphology and internal nutrient composition as influenced by increased temperature and water column nitrate. Estuaries 26, 142-155. doi: 10.1007/bf02691701

Traboni, C., Mammola, S. D., Ruocco, M., Ontoria, Y., Ruiz, J. M., Procaccini, G., et al. (2018). Investigating cellular stress response to heat stress in the seagrass Posidonia oceanica in a global change scenario. Mar. Environ. Res. 141, 12-23. doi: 10.1016/j.marenvres.2018.07.007

Tyberghein, L., Verbruggen, H., Pauly, K., Troupin, C., Mineur, F., and De Clerck, O. (2012). Bio-ORACLE: a global environmental dataset for marine species distribution modelling. Glob. Ecol. Biogeogr. 21, 272-281. doi: 10.1111/j.14668238.2011.00656.x

Viana, I. G., Moreira-Saporiti, A., and Teichberg, M. (2020). Species-specific trait responses of three tropical seagrasses to multiple stressors: the case of increasing temperature and nutrient enrichment. Front. Plant Sci. 11:1696. doi: 10.3389/ fpls.2020.571363

Wang, S. Y., and Zheng, W. (2001). Effect of plant growth temperature on antioxidant capacity in strawberry. J. Agric. Food Chem. 49, 4977-4982. doi: $10.1021 /$ jf0106244 
Waycott, M., Duarte, C. M., Carruthers, T. J., Orth, R. J., Dennison, W. C., Olyarnik, S., et al. (2009). Accelerating loss of seagrasses across the globe threatens coastal ecosystems. Proc. Natl. Acad. Sci. U.S.A. 106, 12377-12381. doi: 10.1073/pnas.0905620106

Wilkes, R., Bennion, M., McQuaid, N., Beer, C., McCullough-Annett, G., Colhoun, K., et al. (2017). Intertidal seagrass in Ireland: pressures, WFD status and an assessment of trace element contamination in intertidal habitats using Zostera noltei. Ecol. Indic. 82, 117-130. doi: 10.1016/j.ecolind.2017. 06.036

York, P. H., Gruber, R. K., Hill, R., Ralph, P. J., Booth, D. J., and Macreadie, P. I. (2013). Physiological and morphological responses of the temperate seagrass Zostera muelleri to multiple stressors: investigating the interactive effects of light and temperature. PLoS One 8:e76377. doi: 10.1371/journal.pone.007 6377

Zimmerman, R. C., Smith, R. D., and Alberte, R. S. (1989). Thermal acclimation and whole-plant carbon balance in Zostera marina L. (eelgrass). J. Exp. Mar. Biol. Ecol. 130, 93-109. doi: 10.1016/0022-0981(89)90197-4
Conflict of Interest: The authors declare that the research was conducted in the absence of any commercial or financial relationships that could be construed as a potential conflict of interest.

Publisher's Note: All claims expressed in this article are solely those of the authors and do not necessarily represent those of their affiliated organizations, or those of the publisher, the editors and the reviewers. Any product that may be evaluated in this article, or claim that may be made by its manufacturer, is not guaranteed or endorsed by the publisher.

Copyright (c) 2021 Beca-Carretero, Azcárate-García, Julia-Miralles, Stanschewski, Guihéneuf and Stengel. This is an open-access article distributed under the terms of the Creative Commons Attribution License (CC BY). The use, distribution or reproduction in other forums is permitted, provided the original author(s) and the copyright owner(s) are credited and that the original publication in this journal is cited, in accordance with accepted academic practice. No use, distribution or reproduction is permitted which does not comply with these terms. 